\title{
Review Article \\ Modeling and Recognizing Driver Behavior Based on Driving Data: A Survey
}

\author{
Wenshuo Wang, Junqiang Xi, and Huiyan Chen \\ School of Mechanical Engineering, Beijing Institute of Technology, Beijing 100081, China \\ Correspondence should be addressed to Junqiang Xi; xijunqiang@bit.edu.cn \\ Received 22 November 2013; Accepted 26 December 2013; Published 10 February 2014 \\ Academic Editor: Rongni Yang
}

Copyright ( 2014 Wenshuo Wang et al. This is an open access article distributed under the Creative Commons Attribution License, which permits unrestricted use, distribution, and reproduction in any medium, provided the original work is properly cited.

\begin{abstract}
In recent years, modeling and recognizing driver behavior have become crucial to understanding intelligence transport systems, human-vehicle systems, and intelligent vehicle systems. A wide range of both mathematical identification methods and modeling methods of driver behavior are presented from the control point of view in this paper based on the driving data, such as the brake/throttle pedal position and the steering wheel angle, among others. Subsequently, the driver's characteristics derived from the driver model are embedded into the advanced driver assistance systems, and the evaluation and verification of vehicle systems based on the driver model are described.
\end{abstract}

\section{Introduction}

Modeling and recognizing human driving behavior have been of interest to researchers from many different disciplines like psychology, physiology, and ergonomics for more than half a century. Great progress has been made from the numerous specific studies on the various aspects of human physiology and psychology by capturing biological data. Driver model research has been made from the perspective of vehicle dynamics application [1] and human factors. Output parameters of driver models are usually steering wheel angle/torque, acceleration or brake pedal position/pressure, and the gear shift position. Driver model can be applied to (1) vehicle dynamics [1] including vehicle component design, vehicle dynamics analysis, overall vehicle stability analysis, and design of onboard controls; (2) intelligent transport systems (ITS) [2-4] including simulation of traffic flow based on the control theory models of driver behavior and modeling driver's risk taking behavior (3) driverless vehicle systems $[5,6]$. This paper aims to present the methods of recognizing driver's characteristics or modeling driver's driving behavior/skill/state from the perspective of driving data in detail, such as vehicle velocity/acceleration, throttle/brake position, and lateral acceleration.
It is commonly known that driving a car is a complex and dynamic task requiring drivers not only to make accurate perceptions and cognitions about information pertaining to the driver's own driving skill, driver state, vehicle performance, and traffic, but also to process all these information at a high rate of speed. Hence, Liu and Salvucci [7] have pointed out that driver models should take into account the characteristics of both high-level cognitive processing and low-level operation controlling.

Modeling human driving behavior and recognizing driver characteristics are necessary to relieve the driver's workload and improve the reliability and amenity of active vehicle safety systems, for example, collision detection and avoidance systems, and road departure warning systems. However, these active safety systems were designed based on an average of driver performance and rarely takes the individual driver's characteristics into consideration. Thus, even though average drivers can benefit from these systems, individual or special groups of drivers such as novices or the elderly might not be able to take advantage of them as effectively. If the characteristics of driver behavior can be accurately recognized and applied to dynamic vehicle systems, the vehicle might be personalized and therefore made intelligent. 
Recognizing driver characteristics is by itself not a simple task with the other requirements of active vehicle safety and comfort of vehicle adding to the complexity. Many active safety systems, such as the automatic braking system (ABS), lane departure warning system, acceleration slip regulation (ASR), and various human-friendly vehicle control systems like adaptive cruise control system, lane-keeping assistant system, have been invented over the years. Information about the driver's driving skill can be used to adapt vehicle control parameters to facilitate the specific driver's needs in terms of vehicle performance and active safety [8]. According to the different objectives set by the various tasks which can be regarded as these actions performed with the help of those functions such as steering, speed control, gear shifting, interpreting the road ahead, and navigation, driving skill can be defined in many ways $[9,10]$. Different tasks require different driving skills. To win a race, excellent driving skills are required. On the other hand, to drive a car from point $A$ to $B$, the driver only needs to obey traffic rules with minimal skills involved.

Generally speaking, modeling and recognizing the driver behavior or driving skill/state can be classified into four steps.

(i) Modeling Driver Behavior. The model structure can be established and parameters of the driver model can be identified based on human driving behavior, which might be classified roughly into three cases: parameter identification, nonparameter identification, and semiparameter identification.

(ii) Recognizing the Characteristics of Driver Behavior. After driver model has been determined, the driver behavior or driver's driving skill should be characterized. Here, many driving tasks or situations (such as car following, lane change, collision avoidance, etc.) are described with numerous mathematical methods adopted.

(iii) Evaluating and Verifying Based on the Driver Model. The objectives of identification followed by the modeling of driver behavior are meant to improve the performance of vehicle dynamics and to design more intelligent driver systems. Therefore, the efficiency of the driver model needs to be evaluated and verified, especially in the field of handling quality and driver assistance systems.

(iv) Embedding Driver Characteristics into the Advanced Vehicle Systems. Producing more intelligent vehicle-driver systems is always the engineer's ultimate goal during the design process. Consequently, driver assistance systems that can timely and accurately detect and predict the driver's attention and seamlessly integrate with the driver's characteristics are crucial.

Based on this, the following sections have been arranged in the order of the aforementioned points.

\section{Identification of Driver Model}

Human driving behavior is extremely complex and contains the human characteristics of nonlinearity, uncertainty, randomness, and so forth. Recently, a large number of articles about modeling driver behavior or recognizing driver model have been published [11-13] from the control point of view. Driver modeling is the simplification of the human driver with logical graphic and equation and so forth and can represent the basic characteristics of human driver like time delay and physical characteristics. Generally speaking, the goal of the driver model is to accurately imitate the driver while accomplishing some assigned tasks, which include two basic parts: longitudinal control (e.g., speed) and lateral control (e.g., steering angle).

The driver model has uncertainty and nonlinear characteristics, but when it comes to certain driving tasks like car following, the structures of the driver model can be determined. According to the certainty and uncertainty of the driver's model structure, the identification methods can be roughly categorized into three aspects from the perspective of pattern identification: parameter identification, nonparameter identification, and semiparameter identification.

2.1. Parameter Identification. During driving, car-following behavior is not uncommon. For example, when a driver is driving a car during rush hour on a highway, the driver may attempt to adjust the vehicle's velocity and its distance by a compromise between the urge to minimize trip duration and to maximize safety. Therefore, car-following models need to be developed in order to enhance traffic safety, and a great deal of car-following models (i.e., Gazis Herman Rothery (GHR) model, safety distance or collision avoidance models (CA), linear (Helly) models, psychophysical or action point models (AP), and fuzzy logic-based models) are presented and discussed in detail in [14]. However, the question of how to recognize the parameters of these driver models from the perspective of system identification under the condition that their structures have been established still remains open.

After one model structure has been prescribed, that is, the model can be shown by a function, and the number of parameters might be finite and fixed, then, the parameter identification techniques can be used to figure out the parameters of the model based on the experiment or simulation data. To address the issue of uncertainty in the driver model structure, Chen and Ulsoy [15-17] have conducted many studies in relation to (driver) model uncertainty including structured uncertainty (e.g., parametric uncertainty) and unstructured uncertainty (e.g., additive uncertainty due to unmodeled dynamics). In [16], while considering the uncertainty both within individual driver and across different drivers, the uncertainty modeling of driver steering control behavior is addressed, and the driver model is treated as a black box, wherein the input and output are lateral deviation from the centerline of the road $\left(y_{\mathrm{dev}}\right)$ and the steering wheel angle $(\delta)$, respectively. Chen and Ulsoy pointed out that the driver model structure considers the uncertainty characteristics, but model selection is dependent on the real driver behavior and the examination of experiment data [15], thus allowing some unstructured or uncertain aspect of driver behavior to be replaced by specific structuralization elements [18] as follows. 
(i) Permissibility or Admissibility. It can also be called the complacency component of driver behavior [15, 19]. One example is the driver keeping his steering command constant when the required change in steering angle is small. This characteristic is illustrated by Figure 1(a).

(ii) Physical Limitations. Most information collected depends on the vision, vestibular, tactile, and auditory perceptions of the driver. However, by reason of human limitations (Figure 1(b)), the driver might be insensitive to some subtle changes. For instance, the driver may be unable to perceive changes in speed when the linear acceleration of the vehicle is lower than $0.005 \mathrm{~g}$ [18].

(iii) Transport Time Delay. There is a possibility of response time being different between individuals; for example, aged drivers might spend more time to brake or steer the wheel than younger drivers. The delayed in obtaining the information before starting to action can be replaced by Figure 1(c).

In [13], two new mathematical models (i.e., an optimal controller model and the "look-ahead model") of driver behavior in a single-lane car following situations were developed and identified using the Fletcher-Powell-Davidon (FPD) algorithm by Burnham et al. in 1974.

In many studies of parameter identification or driver behavior modeling, the ARM [19-21], NARMAX [15], and ARMAX [17] methods are usually selected to establish driver model structures to determine the parameters of the prescribed driver model by using experiment or simulation data.

In [22], to design an ACC controller suitable for driver behavior characteristics, three drivers' longitudinal behavior models including linear regression models, state-space models using subspace-based identification, and behavioral models are identified and implemented by using the collected data with the inputs being the space headway and velocity and its differential, the outputs being throttle angle and brake pressure.

In [23], to develop a driver model of curve driving, both the driver's steering control law (Figure 2(a)) and the vehicle-driver model (Figure 2(b)) are presented, wherein three parameters are used for characterizing the driver behaviors, namely, aim point distance $L_{a}$, response delay $T_{k}$, and steering angle $W$. With the aim of recognizing the parameters of driver model, two targets are prescribed: (1) to decrease the lateral deviation between the actual vehicle position and the driver's desired path as much as possible, and (2) to enable the path realized by the driver-vehicle model to be as similar as possible to the path realized by a real driver. With the aforementioned targets in mind, two simulative scenarios have been designed: the double-lane change maneuver (designed by the Standard No. ISO/TR 3888: 1975) and the driving reaction to wind gust. From the paper, we know that the driver model can be identified and this method can be used to research and evaluate the stability of the driver-vehicle systems, as well as make a combination between the vehicle dynamics properties and the individual driver characteristics. Similar to [23], to describe driver behavior more accurately and to simplify driver models, Saleh et al. [24] developed a cybernetic driver model of lane keeping from the control point of view by adopting the visual, haptic, and kinaesthetic perception and neuromuscular dynamics. The inputs of the driver model are near/far angles, steering angle, and steering force feedback, with the output being steering wheel torque. Subsequently, the driver model can be presented by the state-space structure using the following equation with $\tau_{p}$ being the input delay:

$$
\begin{gathered}
\dot{x}(t)=A x(t)+B u\left(t-\tau_{p}\right), \quad x\left(t_{0}\right)=x_{0}, \\
y(t)=C x(t)+D u(t) .
\end{gathered}
$$

The newly developed cybernetic driver model of lane keeping is simpler and can be easily embedded into the driver assistance system.

However, due to the complexity and uncertainty of driving situations, structures of driver model and targets of driver's choice might vary. Thus, with the aim of building a driver model applicable to a wide range of situations, further research about driver model, advanced mathematical methods, and advanced control theory could be done. For example, more nonlinear mathematical models can be used for characterizing the nonlinear driving behavior.

2.2. Nonparameter Identification. If parts of the driver model structures are uncertain and unstructured and cannot be replaced by the abovementioned elements, then these parts should be treated as a black box and identified by using the nonparametric system identification techniques, such as frequency response analysis (FRS) [25], spectral analysis, and estimating the disturbance spectrum.

In the case of nonparameter identification, the Fourier Coefficient Method (FCM) $[8,26]$ has been used to recognize driver behavior and driving skill.

With the purpose of characterizing and recognizing a driver's limit-maneuver handling behavior, the discrete fourier transform coefficients (DFTC) of steering wheel angle are treated as the discriminant features in [8], and the $N$-point DFTC of steering wheel angle is given as

$$
X_{k}=\sum_{n=0}^{N-1} x_{i} e^{(-j(2 \pi i / N) k n)}, \quad k=0,1,2 \ldots, N-1 .
$$

In [25], to recognize the parameters of a multiloop carfollowing model structure (Figure 3 ) that has only one direct forcing function, the driver transfer function can be identified by using frequency domain identification (FDI) methods.

$U(\omega)$ and $V(\omega)$ are relative velocity and acceleration pedal position, respectively, and can be collected during the experiment. The transfer function $H_{d}^{l}$ of a driver's carfollowing behavior can be identified by the spectral analysis techniques:

$$
\widehat{H}_{d}^{l}(\omega)=-\frac{\widehat{S}_{d u}(\omega)}{\widehat{S}_{d v}(\omega)} .
$$

To find a realistic control theoretic visual driver model of curve driving, the model structure should make the model 


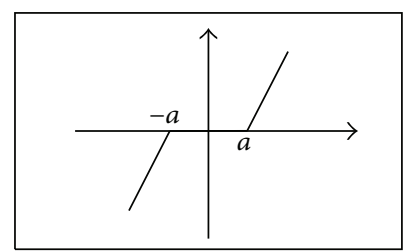

(a)

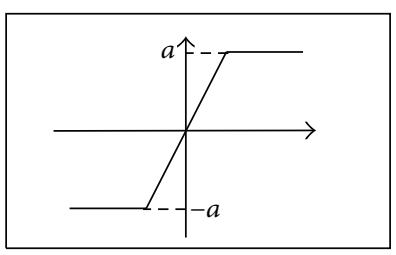

(b)

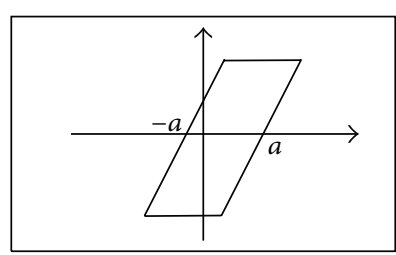

(c)

FIGURE 1: The structuralization elements of driver characteristics: (a) the permissibility, (b) the limitations, and (c) the transport time delay $[15,18]$.

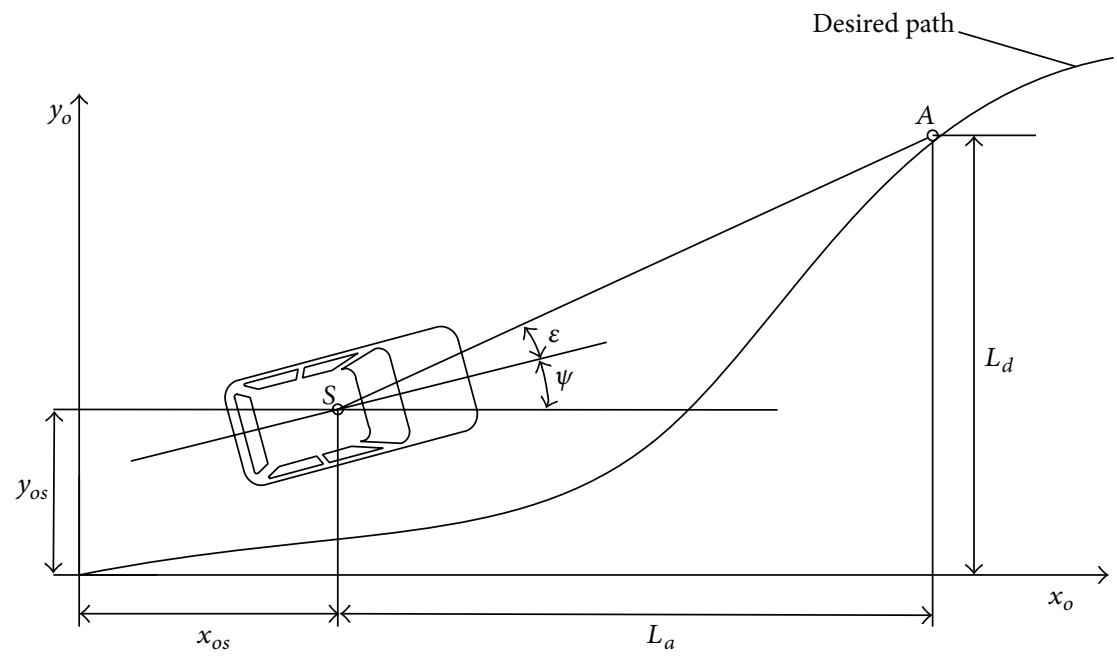

(a)

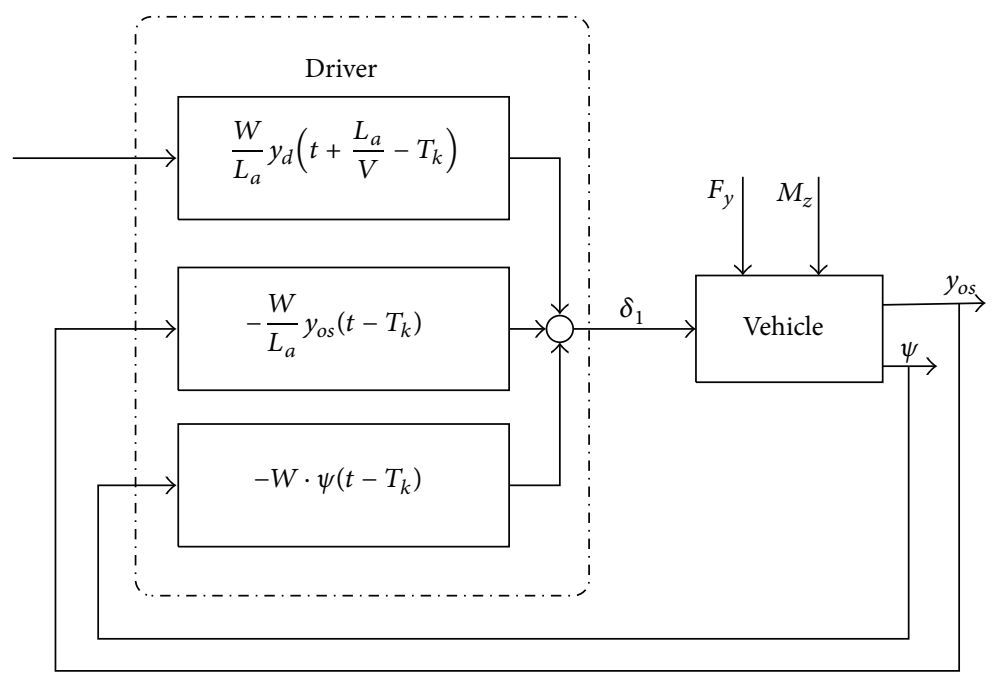

(b)

FIGURE 2: The diagram representing driver-vehicle steering control systems: (a) driver's steering control law, and (b) the vehicle-driver model [23].

parameters to be identified and estimated as accurately as possible. In [26], many models were evaluated and simulated and, if possible, frequency response function was identified using two system identification methods, namely, FCM and ARMAX method.
A car-following model was developed and identified by Wakita et al. [27, 28] using collected driver's behavior signals such as the positions of throttle/brake pedal and vehicle velocity collected via the driving simulator, as well as two different identification models and features. One 


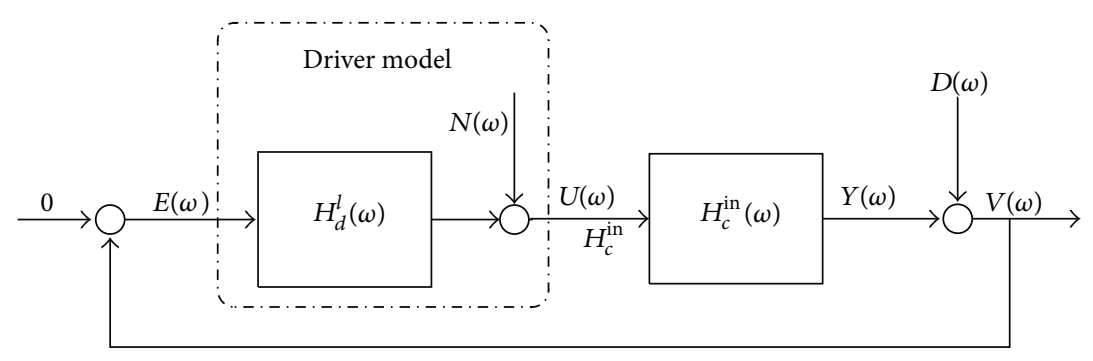

FIGURE 3: The modified driver model suitable for FDI with only one forcing function $D(\omega)$ [25].

is the stimulus-response model (physical dynamic model) assuming that individual driver's personality can be directly characterized by using model parameters; the other is the nonparametric model based on the statistical pattern techniques. Comparing the parametric models with the nonparametric models, the results show that (1) nonparametric models are better than the parametric models, (2) driver's signals (e.g., gas pedal pressure, brake pedal pressure, and steering angle) are more efficient than the environment and vehicle signals (e.g., velocity, acceleration, and engine speed). The nonparametric model shows promising result in [28].

Based on Wakita's conclusions, a nonparametric model with a Gaussian mixture model (GMM) was developed and identified by Miyajima et al. [29], based on the cepstral features of individual driver by using the spectral analysis of driving signals like gas and brake pedal pressures. In this model, the GMM is used to characterize the distributions of features vectors of cepstral coefficient of each driver with the expectation maximization algorithm adopted to estimate the parameters.

It is well known that driving situations (e.g., traffic factors and driver state) are not invariable when driving. For this reason, the driver's model structures and parameters cannot be prescribed. To characterize driving behavior in the case of steady-state and transient car following, a new nonparameter identification model combining the conditional evolving theory with the probabilistic model is developed by Filev et al. [30].

2.3. Semiparameter Identification. Although parameter and nonparameter identification methods have their own merits of identifying different systems as well as having their own operation range. For instance, the former method might have less stringent input or output requirements, but it needs to select a set of candidate driver models which require a known forcing function; however, the latter method suitable for the nonparameter model and black-box model only takes into account the relation between input and output, but ignores the inner state-variables.

To overcome the disadvantages and inherit the merits of both of them, a concept called semiparameter identification is proposed in this part. For example, the nonparameter and parameter identification method are combined together [25] to realize the objective that using only one forcing function to recognize the multiloop model. This method can be treated as semiparameter identification for the entire system identification.
The flow diagram of driver's model identification including parameter identification, nonparameter identifications, and semiparameter identification can be illustrated by Figure 4.

As seen from the above mentioned cases, any type of driver behavior can be modeled and identified using the parameter or/and nonparameter identification techniques, and most of them are based on the linear invariable and offline model. In [11], a real-time identification method of driver's steering manipulation model has been proposed and validated by using driving simulator experiments and the actual driving tests. To exploit the vehicle sensors utter mostly, the yaw rate, the steering angle, and vehicle's velocity are used as collected data, because these sensors have already been installed in a production vehicle.

From the perspective of lateral driving, to control the parameters of steering and lane-keeping behavior effectively, as well as to distinguish the variations in driving performance, in [12], this paper investigates the abilities of two common driver models. One model is based on the human driver visual perception with the input being the deviation angles between vehicle heading and the directions of experimentally determined preview two-points; the other is based on the lane-keeping task with adopted the vehicle lateral deviations and steering wheel angle as input and output, respectively. The preview point model and the lateral offset model can be denoted by $G_{\text {lat }}(q, \theta)$ and $G_{\text {pre }}(q, \theta)$, respectively, where $G_{\text {lat }}(q, \theta)$ and $G_{\text {pre }}(q, \theta)$ can be described as a second-order rational functions of $q$ and $\theta$ :

$$
G(q, \theta)=\frac{B(q, \theta)}{A(q, \theta)}=\frac{b_{1} q^{-1}+b_{2} q^{-2}}{1+a_{1} q^{-1}+a_{2} q^{-2}} .
$$

With identification and validation of the two models, Hermannstädter and Yang [12] have made final conclusions that the output error models is superior to the ARX model in simulation, but this method cannot distinguish the induced driver behavior distinctly. The author pointed out that there might be two reasons resulting in this. On one hand, the second-order model is too simple to correctly describe the characteristics of driver behavior; on the other hand, the uncertainty or unstructured factors (e.g., the signal noise and the nonlinear elements of driver) might make great influence on fidelity and accuracy of the driver model. Therefore, the advanced mathematical methods, such as the stochastic, nonlinear, and fuzzy theories, should be taken into account to develop driver model more accurately. 


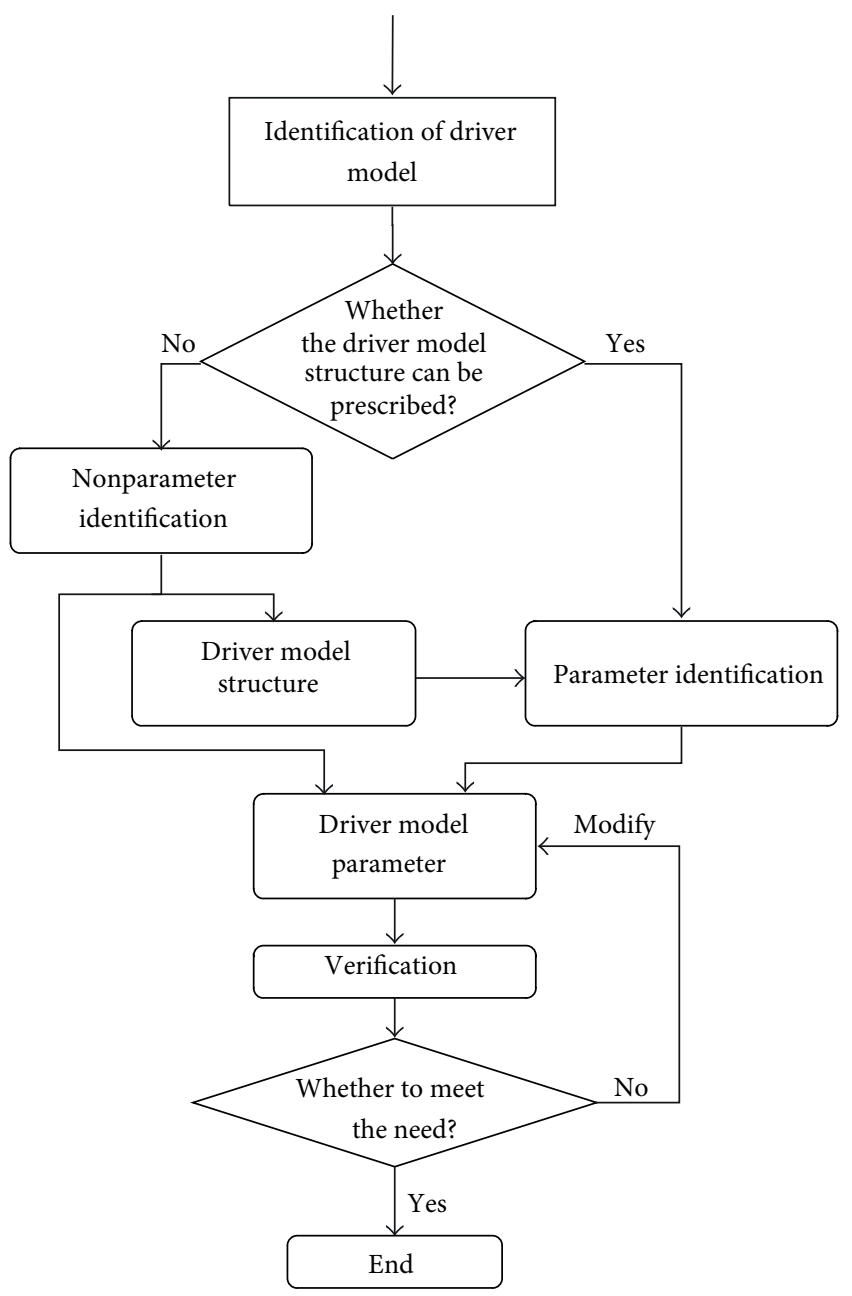

FIgURE 4: The flow diagram of driver model identification.

\section{Identification of Driver Behavior and Skill}

Lots of vehicle dynamic systems and vehicle control systems are designed by engineers, and they generally put their emphasis and interest on the vehicle itself. Recently, highperformance vehicle cannot meet the needs of customers who require more human-friendly vehicle. Thus, human driving skill and characteristics need to be embedded into the vehicle dynamic systems to improve the vehicle's drivability, maneuverability, and fuel economy.

Characterizing driver behavior and skill exactly is crucial to simulating driver behavior and optimizing driver-vehicleenvironment systems. In order to recognize the characteristics of driver behavior and skill, numerous advanced approaches had been applied, as well as more advanced information collecting and processing technology have been introduced into modeling and recognition of driver behavior and skill. In [31], the computer-aided tools including the instrumented vehicles (IVs) and driving simulators (DSs). have been developed.

Human driving characteristics are presented by Macadam [18] from the control perspective in terms of human behavior activities, such as driver distraction, side-tasking, and driver impairments. The author pointed out that humans encompass the characteristics of nonlinearity, time delay, and limitation. Some physical limitations are presented in the realm of human factors by Macadam as followed.

(i) Human Time Delay and Threshold Limitations. Humans can be treated as a nonlinear system with time delay and sense limitations. Time delay consists of dead time resulting from the information processing in the central nervous system and the lag due to the nature of the muscular system, which are different for individual drivers.

(ii) Visual Characteristics. Vision system could not capture the velocity and position information accurately due to the jumplike saccadic response of the eyeball.

(iii) Motion Influences. Due to the influence of vestibular, experience and/or skill level may also play a crucial role in a human-vehicle system.

(iv) Auditory Information. Auditory information may be more useful under high workload conditions, and in general it can be treated as redundant information.

(v) Tactile and Haptic Information. Tactile and haptic information (e.g., steering wheel torque, the pedals position) conveyed through the steering wheel and throttle or acceleration pedals, but the fidelity of the information has threshold limitations.

Based on the assumption that a driver remains in control most of the time, a method to characterize and evaluate the specified driving skill was developed on the basis of path tracking driving skill by Erséus [9] in driving simulator tests. In these tests, four scenarios are designed as follows.

(i) Curved Cone Track Scenario. According to [32-34], we know that road width and curve radius have great influence on driver's speeds choice that can be treated as driver characteristics. The goal of this scenario was to investigate driver behavior with a focus on the variation of different driver's ability to steer the vehicle, that is, path tracking skill.

(ii) Avoidance Maneuver Scenario. This scenario was used to evaluate the relationship between driver skill and many objective vehicle parameters measured in the moving base simulator at VTI.

(iii) Driver Response Scenario. This scenario was designed and evaluated for the investigation of driver-vehicle characteristics when following a movable reference line, that is, line jump scenario.

(iv) Curving Road Scenario. Curving road scenario was designed in order to evaluate objective parameters of driver's driving skill when he or she drives on a normal curving road.

Erséus thought that there are some limitations in [9]. (1) This research just focused on the path tracking driving skill of drivers holding a Swedish driving license. (2) All the results are concluded based on the research about behavior 
characteristics of the group, not the individual driver. (3) The scenarios designed are too few to demonstrate that whether tracking driving skill is the same with other driving skills or not. Thus, it is necessary to make further research about other types and characteristics of individual/group driver's driving skill in the same scenario, but different scenario parameters.

Angkititrakul et al. [35] developed a stochastic driverbehavior model that can characterize individual driver better than universal models in both short-term and long-term predictions by using the observed driving data based on Gaussian mixture model (GMM). Nevertheless, there are some disadvantages with the GMM, mass data should be collected and processed in-time in order to establish individual driver models more accurately. Then, to recognize individual and general driver's characteristics, Angkititrakul et al. [36] presented an improved driver-behavior model which involved both of them, and the patterns of individual and general driver styles are modeled by using Dirichlet process mixture model (DPM) and GMM, respectively. The result shows that the integrated model can better represent both observed and unobserved individual driver's behaviors.

It is commonly known that driving behavior/skill/styles are influenced by numerous factors, such as the driver's physiology/psychology, driving environment, and traffic conditions. The precise-driver model should take these factors into consideration, but it is not practical to collect and process so much data in-time. In addition, driver's model structures and parameters are uncertain in most common conditions. Therefore, a stochastic evolving real-time identification method was introduced in [30], and a new driver model was developed under the steady-state and transient car-following situation. The results show that this driver model is able to characterize driver's dynamic behaviors effectively in the uncertain driving situations.

Lin et al. [37] propose key parameters in a dynamic driver model to characterize driving skill. The general overview of [37] is illustrated in Figure 5. In this approach, the driver model is dynamic so as to mimic human driver outputs, and using an extensive set of driver model parameters the driver skill level is categorized into three levels: lower, typical, and expert. This model-based approach depends heavily on the validity and fidelity of the mathematical driver model.

In [8], Zhang et al. compared the utility of various pattern-recognition algorithms, including multilayer perception artificial neural networks (MLP-ANNs), decision tree, and support vector machines (SVMs), based on the coefficients of the discrete Fourier transform (DFT) of the sensor information (e.g., steering wheel angle, yaw, and lateral acceleration) getting from the driving simulator. The experiment results show that the DFT coefficients of the steering wheel angle not only can be applicable to discriminating the expert drivers from typical or low-skilled drivers, but also could be used as the discriminant features. Zhang et al. addressed this problem in their proposed pattern-recognition approach [8] for driving skill characterization. This approach is based on the theory that there are strong correlations between the driver's behavior and vehicle response. In this approach (Figure 6), the driver-modeling step is skipped and the relationship between driver's overt behavior and the driver's driving skill is directly build. While both of these proposed an approach for driving skill characterization in different driving courses based on the driving simulator, it is hard to explain which course parameters (e.g., the course curve radius) could characterize driver's driving skill best.

In [19], a recognition method of steering behavior (e.g., lane keeping, lane changing) was presented and a new arithmetic was developed to improve the awareness of driving safety by using sequential labeling method based on boosting framework. To develop a discrimination model of lane change behavior recognition algorithm (boosting algorithm), four features are focused on: velocity, steering wheeling angle, moving variance, and moving standard deviation. One main result of these experiment data is that the threshold values of lane keeping and lane change behavior are different depending on the vehicle's velocity even if the moving standard deviation of steering wheel angle is the same value.

In [20], Pilutti and Ulsoy presented an online identification approach of driver state that is a desirable element of many proposed active safety systems. In this approach, an ARX model is allowed to describe the relationship between vehicle lateral position $(y)$ and steering wheel angular position $(\delta)$ based on driving lane-keeping task. In the model, $y$ and $\delta$ are the input and output, respectively, with an ARX structure as the candidate model structure:

$$
A(q) y(t)=B(q) u(t-n k)
$$

where $y(t)$ is the driver model steering position output $(\delta)$, and $u(t-n k)$ is the delayed driver model input. Then, in [38, 39 ], the approach was applicable to collecting data from 12 2-h highway driving runs conducted in a full-vehicle driving simulator. In particular, in [38], the authors pointed out that there were five aspects of shortages in this approach: model structure inadequacy, nonlinear effects, poor model fits, trends masked by variations in parameters, and alternative approach.

Driver behavior encompasses the characteristics of dynamic, randomness, and nonlinearity, as well as obeying certain distribution. The complex mapping from sensory input to driver's action output might be strongly nonlinearity in nature; hence, the traditional control methods like PID control are unable to simulate human-driver-vehicle system actually. To overcome this problem and to improve the validity and fidelity of driver model, most of stochastic, nonlinear, and fuzzy theories (e.g., Hidden Markov Model system (HMMs), Hierarchical Hidden Markov Model (HHMM), autoregressive HMM (AR-HMM), nonlinear regression models, and the neural networks and fuzzy systems) have been used to recognize and predict driver behavior (see [40-45]).

Rich in mathematical structure, HMMs are powerful parametric models which have been applied extensively in the area of stochastic signal processing. To overcome (1) dynamic and/or (2) stochastic of driver model in nature, Pentland and Andrew [43], Nechyba and Xu [44] propose a driver model using HMM, and then the fidelity of the driver model is verified.

Drivers collect information of the vehicle or environment such as vehicle position, road profile, and pedestrians mainly 


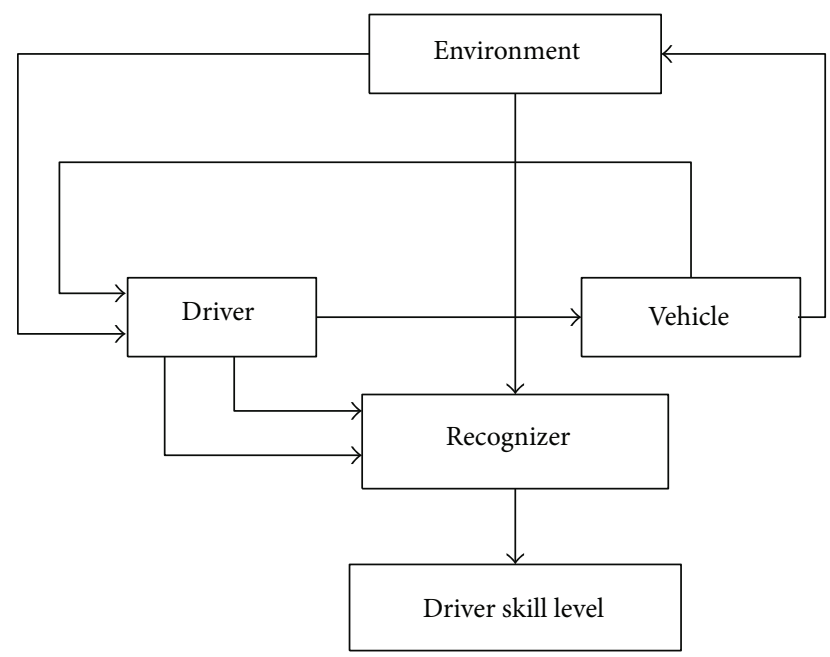

FIGURE 5: Model-based approach for driver skill characterization [37].

via visual system during driving. Macadam [18] has shown that vision ranks the top among the primary sensory (vision, vestibular and kinesthetic, tactile, and auditory) channels used in driving environment. With the visual information as a key consideration, Liu and Salvucci [7] described the application of Markov Dynamic Models (MDMs) in the field of modeling and prediction of driver behavior based on the assumption that driver's visual scanning behavior can be treated as another source of driver's state information.

As stated by aforementioned driver characteristics, it is obvious that driver-vehicle systems have the same characteristics as humans in nature. In recent years, the vehicles installed active safety systems are not uncommon in modern car, but most of them are designed by engineers who rarely take into account the human factor during the design process. Thus, modeling human-vehicle systems allowing for clarifying the relation between driver and driver assistance systems can facilitate the operating mode transitions. Kuge et al. [45] have proposed a recognition method of driver behavior by adopting HMMs to characterize and detect driving maneuvers, and then it was applied to the framework of a driver's behavior cognitive model. The authors put emphasis on information processing models of human drivers with using them to detect and recognize model-based HMMs. This paper demonstrates that (1) HMMs can be used to recognize the frame of driver model based on driver's lane change behavior; (2) an active vehicle safety system embedded with driver model can be developed. Although HMMs have some advantages of both recognizing certain driver behavior and mapping the relation between driver behavior/state/skill and vehicle responses (e.g., yaw, yaw rate, vehicle velocity, and acceleration), some questions still remain open as to its validity of general application in research.

Sekizawa et al. [21] pointed out that stochastic and nonlinear characteristics of the human driver could be expressed as much as possible by the abovementioned models, but there are two shortages in them. (1) The aforementioned models are often too complicated to recognize model parameters

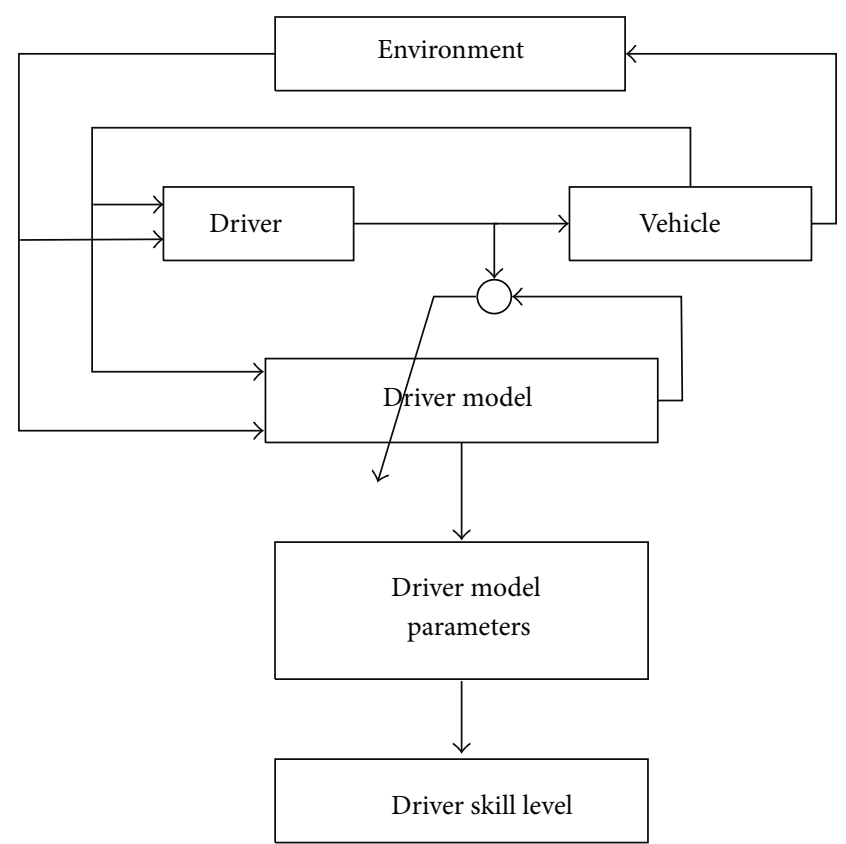

FIGURE 6: Pattern-recognition approach.

rapidly and accurately, and (2) this, in return, makes it impossible to understand the physical behavior; it is often found that a driver appropriately switches between certain simple primitive skills instead of adopting a complex nonlinear control law. To formally address these shortages, in [21], modeling and recognition of driver behavior were developed based on a stochastic switched autoregressive exogenous (SSARX) model (Figure 7). The SS-ARX model is applied to characterizing driver's collision avoidance behavior at the instance when the preceding vehicle is brought to a sudden halt and the examinee is looking away from the road.

With the aim to simulate driver's collision avoidance behavior, three kinds of driving information, such as range between cars, range rate, and lateral displacement between cars, are collected, and the output value is also specified as steering amount. This experiment result shows that each driver's characteristic is unique; in particular, large variations are observed between driver behaviors with respect to the lateral displacement between cars and steering profiles.

Compared to the HMM or neural network model (NNM), the SS-ARX model has some advantages over both of them. First, the SS-ARX model can provide the information with extraction of driving primitives, but the HMM cannot. Second, when it comes to the discrete modes, the SS-ARX may show unique advantages over the standard HMM. Third, NNM can obtain the parameters of driver characteristics; however, the significance of them is not clear. Therefore the SS-ARX model can present the part from the control perspective with the switched controlled mechanism.

Since the piecewise polynomial (PWP) model or piecewise linear (PWL) model [46] includes both continuous behavior given by polynomials and discrete logical conditions, it can be regarded as a class of hybrid dynamical system (HDS). A modeling strategy of human driving behavior based 


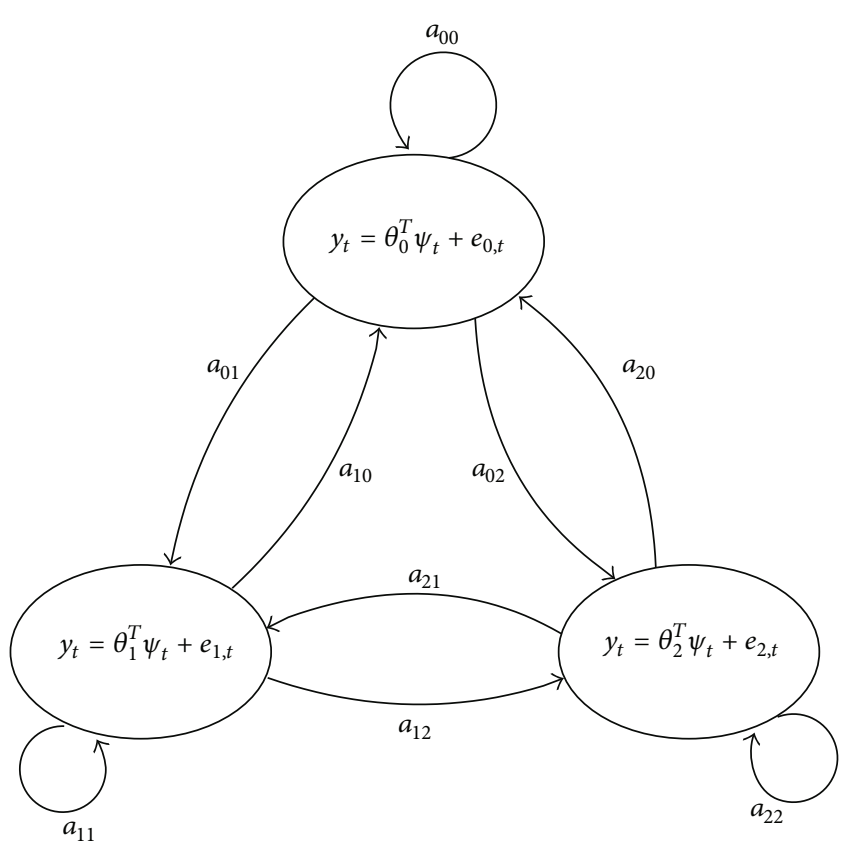

FIgURE 7: The SS-ARX model (three modes) [21].

on the controller switching model with focus on driver's collision avoidance maneuver was presented by Kim et al. $[47,48]$. This model was expressed by PWP or PWL model, and the driving data (acceleration, braking, steering, etc.) are collected by using a three-dimensional driving simulator (3D-DS) based on CAVE. In this model, the driver's collision avoidance maneuvers are divided into four piecewise modes: the first period of avoidance, the second period of avoidance, the first period of recovery, and the second period of recovery. Subsequently, the parameters of every piecewise mode were identified using the mixed integer linear programming (MILP) techniques.

In [49], Michon held that driver model tasks should probably best be further classified into three hierarchies of skill from the driver's control perspective.

(1) Strategical (Planning) Level. This level can be treated as decision-making level from long-term perspective, such as the choice of trips goals, route, and driving model. For instance, taking cosiness and fuel-saving into consideration, drivers will choose to drive on roads in good traffic conditions.

(2) Tactical (Maneuvering) Level. In this level, the controlled action patterns, such as obstacle avoidance and overtaking, should be considered, and this level only takes up a few seconds.

(3) Operational (Control) Level. This level is defined from the point of view of control including steering, braking, and accelerating control. The automatic action patterns can be derived from this level.
Based on the three levels, a hybrid dynamical system (HDS) [50] with two parts (i.e., decision making and motioncontrol) included was proposed and designed by Kiencke et al. in 1998. The HDS can be illustrated by Figure 8, which consists of (continuous) primitive and (discrete) switching driving operations [51, 52]. Since the HDS was proposed, most researches of HDS (see, [47, 53-60]) have been made. In [51], a driving behavior model was developed based on the HDS, and a piecewise ARX (PWARX) model was established using driver's sensory information (e.g., the range between vehicles, range rate, and time derivative of the area of the back of the preceding vehicle) and the output of driver behavior, such as pedal operation. The parameters appearing in the primitive (continuous) and switching (discrete) operations can precisely be identified by using the PWARX model, allowing for the developed model to be used to design the advanced driver assistance systems that can switch between multimodels [53] in the HDS. However, the PWARX model cannot distinguish the overlapping modes explicitly, which is the first step to recognize driver model.

To address the issue mentioned in [51], Okuda et al. [61] have proposed a probability-weighted ARX (PrWARX) model, wherein the probabilistic weighting was given a crucial consideration. The difference between PWARX and PrWARX is that the deterministic partition in the PWARX model is replaced by softmax function:

$$
P_{i}=\frac{e^{\eta_{j}^{T} \cdot \varphi_{k}}}{\sum_{j=1}^{s} e^{\eta_{j}^{T} \cdot \varphi_{k}}}, \quad \eta_{s}=0,
$$

where $\eta_{i}, i=1,2 \ldots, s-1$ is used to represent the probabilistic partition between regions corresponding to each mode. By introducing the probability-weighted concept, the decision entropy can be defined and applied to describe the vagueness in the switching operation, as well as being used as a verification index of the model.

In [62], an approach to recognize driver's driving manipulation skill is presented based on the HDS model. Different from the previous ones, HDS is treated as a hinging hyperplane autoregressive exogenous (HHARX) model in which each continuous submodel deals with its related manipulation model, and meanwhile, discrete model can switch between all submodels. Then, the parameters of HDS were identified by using a mixed-integer linear programming (MILP) method. Lastly, the identification model is embedded into a microcontroller to design an automatic driving system [63] with real-time image collection and processing.

Generally speaking, driving can be considered as a dynamic behavior, and the parameters of driver model might change with driving conditions and driver's psychologi$\mathrm{cal} /$ physiological state. Hsiao [64] thought that the previous methods, such as ARX, ARMAX, and HMM, are mostly based on the linear time invariant (LTI) system, which can only be an approximation of driver behavior for a short time. Therefore, the LTI system may not recognize the timevariant parameters precisely. In response to this issue, a time-varying system identification method (i.e., time-varying ARX) has been developed by Tesheng Hsiao using maximum 


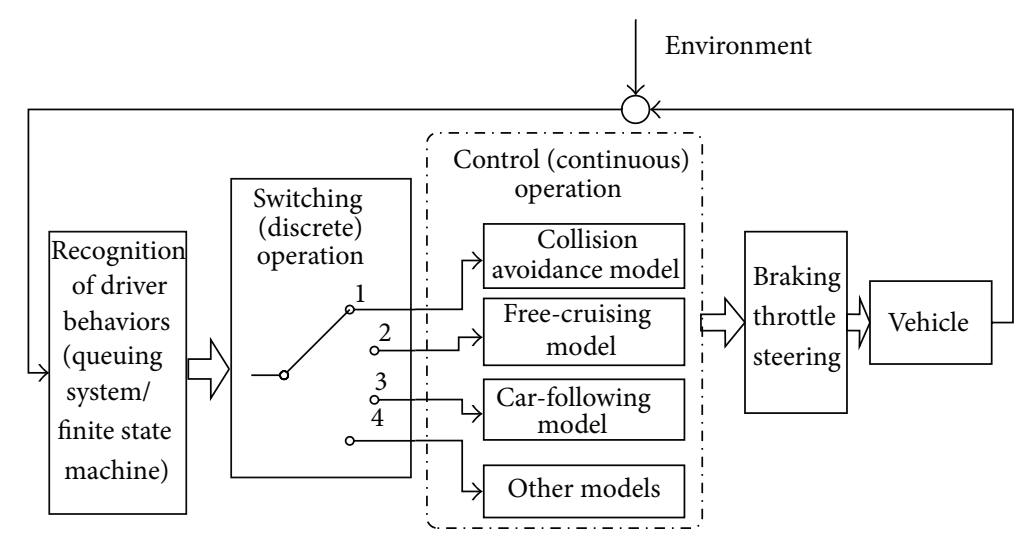

FIGURE 8: Optional multimodels of hybrid dynamical systems (HDS) containing the (continuous) primitive driving operations and their (discrete) switching.

a posteriori estimation, and the model can be described as in the following equation:

$$
y(k)=-\sum_{i=1}^{n_{a}} a_{i}(k) y(k-i)+\sum_{j=0}^{n_{b}} b_{j}(k) u\left(k-j-n_{k}\right)+\varepsilon(k),
$$

where $y(k)$ and $u(k)$ are output and input sequences, respectively, $\varepsilon(k)$ is the process noise, and $a_{i}(k), b_{i}(k)$ are the system parameters required to be identified.

HDS can clearly describe driver model or driving task, and one of its crucial issues is how to recognize the distinct state of driving operation from driver behavior and to determine the number of the state. In [51], the hierarchical clustering method was applicable to estimating the number of state, and then a stochastic piecewise affine (PWA) model was developed by Okamoto et al. [52].

\section{Evaluation and Verification Based on Driver Model}

This section addresses the evaluation and verification of vehicle handling qualities and advanced driver assistance systems based on the driver model from the control perspective.

4.1. Handling Qualities Evaluation. The concept of handling quality is first explained via the field of aerospace engineering [65] and is later applied to land vehicle design and evaluation. Harper [65] discusses the assessment of handling quality characteristics since handling quality deals with more than one element. Accordingly, an airplane and its pilot were represented in order to assess handling quality. In the case of driving a car, the car and its driver are incorporated into the system in the same manner. Therefore, when evaluating the handling quality, the driver should be taken into consideration. Conventionally, handling quality evaluation can be classified into objective handling quality evaluation and subjective handling quality evaluation which is made by observing the dynamic characteristics of the automobile and driver, respectively. A subjective method based on the driver's comments can be used to evaluate the handling quality in a relatively precise way, but this method requires many actual driving tests that include every design parameter. Furthermore, these evaluations might differ between different test drivers. Especially while evaluating each driver's limitmaneuver handling behavior, this is very dangerous for the test-takers.

Since the 1990s, researchers began studying driver steering dynamics models that could replace test drivers. In [66], a control theoretic model of driver steering dynamics is developed and demonstrated to be able to produce driver/vehicle steering responses and compares favorably with those obtained from driver simulations. Using the theoretical model of driver steering dynamics, engineers who may not be experts in manual control are enabled to evaluate handling or maneuverability.

Driving style, behavior, and skill might vary for different drivers causing evaluation criteria to be diverse and in turn sharply reduce confidence of the handling quality indexes. Therefore, it is imperative to define driver characteristics as a criterion for handling objective evaluation. Reference [67] states that a parametric driver model for ISO lane change simulation was developed using the decreasing parameters dispersion method by Carlol et al.

Similar to [67], while making a subjective evaluation of handling quality, a multiloop structure of closed-loop drivervehicle systems including a multi-input driver model was developed by Horiuchi et al. [68]. In this driver model, the inputs are the lateral position error $y_{e}$ and the yaw angle $\varphi$, with output being the steering angle $\delta$. To characterize driver dynamics accurately, the three essential factors of time lag $e^{-\tau s} /\left(T_{1} s+1\right)$, predictive action $\left(T_{L} s+1\right)$, and proportional action $K$ have been taken into consideration. The driver model can be described by the following equation:

$$
\delta=y_{e}\left[K_{y}\left(T_{L y} s\right)+1\right]-\psi\left[K_{\psi}\left(T_{L \psi} s+1\right) \frac{e^{-\tau s}}{T_{1} s+1}\right] .
$$

Subsequently, an analytical approach to subjectively rate handling quality of actively controlled vehicles is discussed and applied to the evaluation of the handling quality of fourwheel steering vehicles. Here, the three principle factors of 
the objective function, that is, the task performance $J_{1}$, the driver's mental workload $J_{2}$, and his or her physical workload $J_{3}$, are considered. The results show that this method not only can be applicable to the prediction of the subjective evaluation of handling quality, but is also able to characterize driver-vehicle systems.

By analyzing the driver's characteristics and incorporating them into the closed-loop road-vehicle-driver test system (Figure 9), a cost function of the handling quality that can be used to estimate the handling quality analytically from the vehicle's dynamics is constructed [69]. Three crucial characteristics of driver handling are obtained with the driver-in-the-loop system by Miura et al. and are (1) the driver model's response to the yaw rate has a strong connection with the evaluation of handling quality, (2) different road conditions such as road radius and profile, could result in the drivers having different frequency responses, and (3) there are no notable differences in how each driver operates between driver and vehicle dynamics even though automobile dynamics are different. Based on the aforementioned characteristics, a benchmark driver model has been built and is applied to the handling quality rates in the absence of the driving experiment.

This approach uses two driver models (i.e., transfer function, $\left.H_{1}(s), H_{2}(s)\right)$ to represent the human control with the inputs $e_{y}$ and $e_{r}$ and the outputs $\delta_{f 1}$ and $\delta_{f 2}$. In Figure 9, $H_{1}(s)$ and $H_{2}(s)$ are the steering responses to the lateral displacement $y$ and yaw rate $r$, respectively. Correspondingly, $e_{y}, e_{r}$ are the deviations between the reference value and the actual value; $P(s)$ is the transfer function of a simple fourwheel nonlinear passenger automobile model applied as a vehicle model.

Because drivers can adapt themselves to the handling characteristics of the vehicle during a driving maneuver, their steering behavior reflects the vehicle's handling characteristics and plays an important role in the evaluation of handling quality. In [70], an approach to evaluate vehicle-handling quality based on steering characteristics is presented; wherein the steering characteristics were identified by a simple driver model using the relationship between the time histories of steering behavior and vehicle motion during lane change. The study presented a closed-loop driver-vehicle system (Figure 10), with $H(s)$ and $P(s)$ representing the driver model and the vehicle model correspondingly. A resulting transfer function of steering angle to lateral position error during a lane change is used as the driver model $H(s)$ with the deviation of lateral displacement $\Delta y=y_{0}-y$ as the input and the steering angle $\delta_{h}$ as the output and can be described as

$$
H(s)=G_{h} \frac{1+\tau_{h} s}{1+T_{h} s},
$$

where the driver steering parameters $G_{h}, \tau_{h}$, and $T_{h}$ are steady-state gain, the derivative term of differential control, and the time constant of the first-order lag, respectively.

4.2. Evaluation and Verification of Driver Assistance Systems. Similar to the evaluation of handling quality, it is necessary to evaluate and verify the DAS with the human driver in the closed loop. Reference [71] presents two newly developed driver models, which are applied to evaluating the impact of ACC vehicles on traffic flow and the effect of a vehicle stability control (VSC) system on possible vehicle roll prevention. One of driver models is the modified Gipps model which is used for evaluating the ACC, whereas the other model is applied to the evaluation of active safety systems based on the adaptive plant inversion concept. In its accompanying paper [72], a longitudinal human driver model (i.e., the modified Gipps model) used for performance evaluation of the ACC system on highway traffic from the microscopic and macroscopic traffic perspective was developed and simulated by Lee and Peng.

The majority of DASs can release drivers from some secondary tasks during driving and improve on safety, comfort, and performance. Notwithstanding, the driver might be confused, annoyed, and distracted, if he or she is sensitive to the monitoring and the excessive detection or frequent warning of DASs. For this reason, a driver model used to evaluate DSAs that can precisely mimic human driving is required so as to consider mistakes committed by human drivers. To achieve this, in $[73,74]$, an errorable driver model (Figure 11) was developed to evaluate both the collision warning and collision avoidance algorithms. The errorable driver model can generate both nominal (error-free) and devious (with error) behavior like in humans. Three common driver mistakes, namely, human perceptual limitations, distractions, and time delay were considered in establishing this errorable driver model.

Numerous driver models can be applied to the vehicle design process and to the evaluation or verification of active safety systems. Most of them are designed with the average (general) or atypical driver in mind and thus are unable to represent individual characteristics. This is illustrated by the fact that the DASs assessed by the universal driver model and its results show that the DASs are suited most for the common driver, but not the individual. In the case of an anxious and impulsive driver, the DASs might give warnings too frequently resulting in the driver getting bored with the DASs and accordingly decrease his or her situational awareness, comfort, and safety and ultimately increase the driver's workload. Hence, a driver model that can effectively characterize individual driving behavior, skill, and styles should be further developed and applied to the evaluation and verification of active safety systems.

\section{The Advanced Vehicle System Embedded with Driver Characteristics}

The ideas previously discussed show that recognizing the characteristics of driver's driving behavior/skill/state are vital to the drivers' safety, vehicle design, fuel efficiency, and vehicle ergonomics. Driving is a complex task which should be executed with several nonlinear subsystems such as the human driver, surrounding vehicles, driving environment, and electronic control systems. It is widely known that a normal/experienced driver can adapt to different vehicle systems and/or driving environments in a short period of 


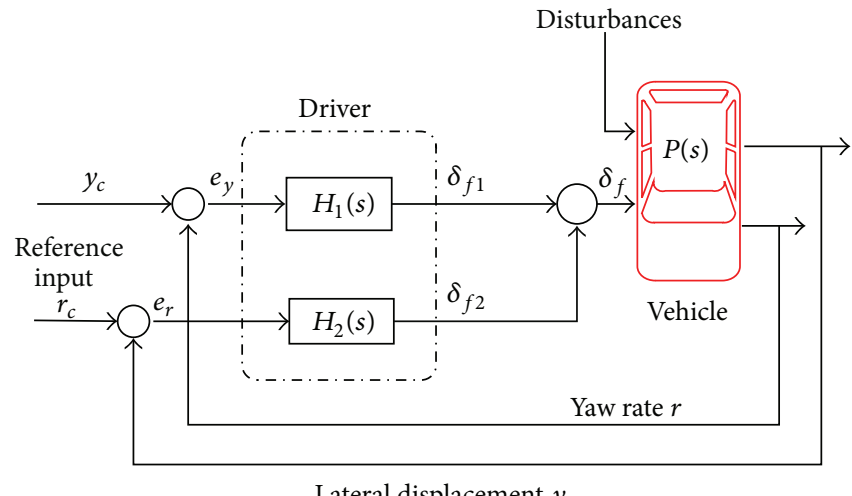

FIGURE 9: Driver-in-the-loop system [69].

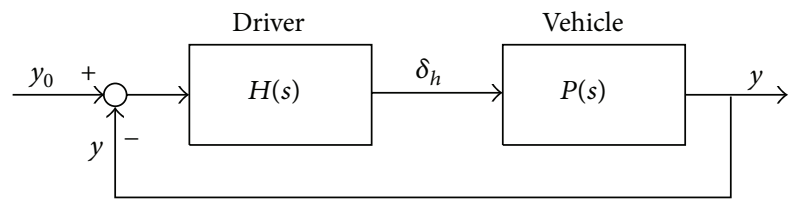

FIGURE 10: Driver-vehicle system.

time by adjusting his or her nerve (neuromuscular) units. The human driver can develop an optimal route by taking into account long or short periods and change the throttle/brake position and steering wheel angle according to the collected real-time information and instantaneously adjust the vehicle's position or velocity and decrease the deviation between reality and expectation. In other words, after perceiving and processing the driving situation, the driver can select process control rules suitable for the situation that allow him or her to manipulate the vehicle's controls in a manner that satisfies the driver's control objectives even in different scenarios.

Most research on how drivers adjust various dynamic vehicle systems to adapt with their driving environments was conducted from the standpoint of psychology and physiology. To lighten the workload of drivers and in turn reduce the occurrences of traffic accidents, the idea of assisting drivers on the road was proposed for which the various driver assistance systems were subsequently developed. Even though the DAS can alleviate the driver's workload, there is still a chance of it having a negative effect on the driver-vehicle systems due to the disharmony or adverse interactions between the driver and the assistance systems under certain conditions; in conventional car systems, most of the DASs are electronic control systems with invariant design parameters. As it is not hard to imagine a novice driver benefitting more from the early intervention of a power-assisted steering system than an experienced driver [8], the intelligent driving assistance system (IDAS) or the advanced driver assistance system (ADAS) was developed. In the IDAS or ADAS, driver characteristics are embedded into both the longitudinal control and lateral control. In [75], Fancher et al. researched on driver-vehicle coordination before proposing the human-centered vehicle system concept which has resulted in many similar theories and models following soon after.

5.1. Longitudinal Control Based on Driver Characteristics. With the rapid development of vehicle and traffic technology, car following has become the most prominent driving task, especially while driving on highways or urban roads during rush hour, with the aim of maintaining a safe and comfortable car-following state for the purposes of mitigating the workload of drivers, reducing the occurrences of traffic accidents, and increasing traffic flow rate. Several DASs have been developed based on driver characteristics from the control perspective such as the adaptive cruise control (ACC), stop and go (S\&G), and forward collision warning/avoidance (FCW/FCA).

Vadeby [76] has studied relative collision safety models together with driver characteristics for ten years. The change in consumer demand is reflected in the main design objectives shifting from power and performance to safety, comfort, and intelligence. Accordingly, researchers have focused more on human characteristics and designed "human-centered" automation or operations that account for the driver's expectations and automation goals. Despite being a sound theory, it is still difficult to describe driving behavior to arrive at a unique optimal multiattribute method for solving problems including designing a "human-centered" controller with these multiple attributes in mind. To address the issue, Goodrich and Boer [77] developed a systematic method that uses a multiattribute breakdown of human and automation goals which has been subsequently applied to the design of human-centered collision and accident avoidance systems (CAAS).

Similar to [78], the human-centered DASs including ACC and FCW were also presented by Fancher et al. [75]. To be human centered, DASs need to take vehicle dynamics into account and match them with the driver's physiological, psychological, and other attributes. Various aspects of human-centered DASs were also discussed, namely, the looming effect, ruler-based and skill-based behavior, the utilization of desired dynamics in controlling the driving process, and braking rules or collision-warning rulers. After 


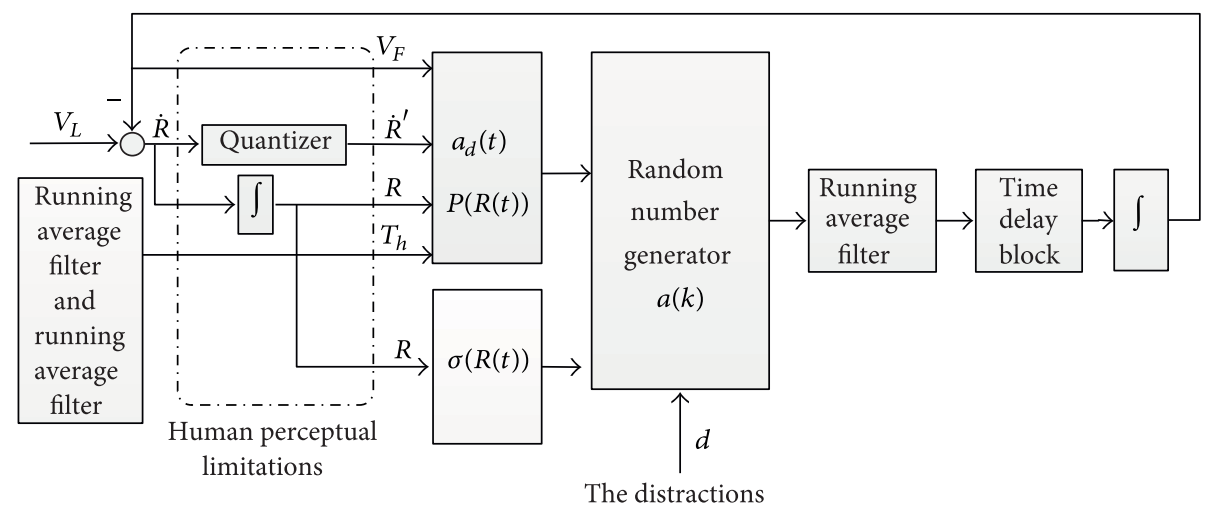

FIGURE 11: The diagram of the errorable driver model $[73,74]$.

which, the collected field-test data and on-road data were used to evaluate and verify the human-centered DASs. This paper makes great progress in explaining when and why a driver makes a braking action. The author also points out that answering the following basic questions about braking might facilitate the development of human-centered DASs. (1) When should braking occur or not occur? (2) What is the control objective when driving? (3) How do drivers do it?

In $[78,80]$, to develop and verify a new automatic advanced vehicle system integrated with human driver characteristics, an instrumented vehicle test bed called the Laboratory for Intelligent and Safe Automobiles-Q45 (LISAQ) was designed, after which, a collaborative approach for developing human-centered DAS (e.g., ACC) was proposed by McCall et al.

Though longitudinal control systems (ACC, FCW, etc.) can be designed with driver characteristics taken into account, some issues still exist. For example, owing to the finite number of techniques and the incompatibility of subsystems, excessive, inaccurate, or contradictory information might be transmitted, causing advanced vehicle systems to disturb, distract, or even overwhelm the driver. In [81], Zheng and McDonald raised the question on whether "drivers' expectations can be matched". They rightly pointed out that at present, no existing ACC system can deal with the full range of complex traffic situations in practice and that humans should act only as a "monitor" in ACC equipped drivervehicle systems. When a driver's expectations are breached, for instance, he or she will make interferential actions between his or her intentions and the ACC's capability. The best DAS system is not the one most capable of following traffic but the system that considers both comfort and safety characteristics the most. In order to improve the compatibility between ACC performance and driver's expectations, a large number of situations have been tested by changing variables systematically such as the parameters of the ACC algorithms, traffic scenarios, and time-headway settings. The results reveal that an appropriate ACC setting capable of meeting a driver's expectations can be found and that the ACC setting most adept in a range of traffic conditions may not necessarily be the most user-friendly.
Road conditions such as road profile and road friction coefficient might have great influence on the driver's characteristics and the driver-vehicle systems. To deal with the problem of rear-end crashes of moving and parked vehicles, Nakaoka et al. [82] conducted further research on forward collision warning systems (FCWs) that took into account road conditions (dry and wet) and individual driver characteristics. Time to collision (TTC) is usually used to evaluate the severity of a forward collision as the host vehicle approaches another vehicle from the front and can be calculated by

$$
\mathrm{TTC}=\frac{X_{l}-X_{f}}{V_{l}-V_{f}},
$$

where $X_{l}$ and $X_{f}$ are the positions and $V_{l}$ and $V_{f}$ are the velocities of the leading and host vehicles, respectively. The formula states that the TTC is related to both the host and leading vehicles; therefore, using it to characterize individual drivers may be unreasonable. In [82], the timing of a driver's braking reaction time is used as a proxy for hazardous level instead of the traditional TTC.

Most driver behavior models applied to the automobile are limited to the single driving task, such as lane keeping and car following. In [79], in order to integrate individual driver characteristics into driver assistance, the state transition feature for individuals are taken into account, more specifically, the five categories involved in longitudinal driving situations of car following, braking, free following, decelerating, and stopping. Longitudinal vehicle dynamics driving data such as acceleration and braking was collected. By classifying the longitudinal driving situations into five parts and adopting the boosting sequential labeling method, the framework for driver-vehicle-environment (Figure 12) can be modeled allowing for the characterization of the driver state, followed by the design of advanced and personalized driver assistance systems.

Reference [83] describes a longitudinal driver model designed to embed individual driver characteristics into an advanced driver assistance system that is mainly applied to simulating throttle and braking operations. In this driver model, a generic model is developed based on the driving data 


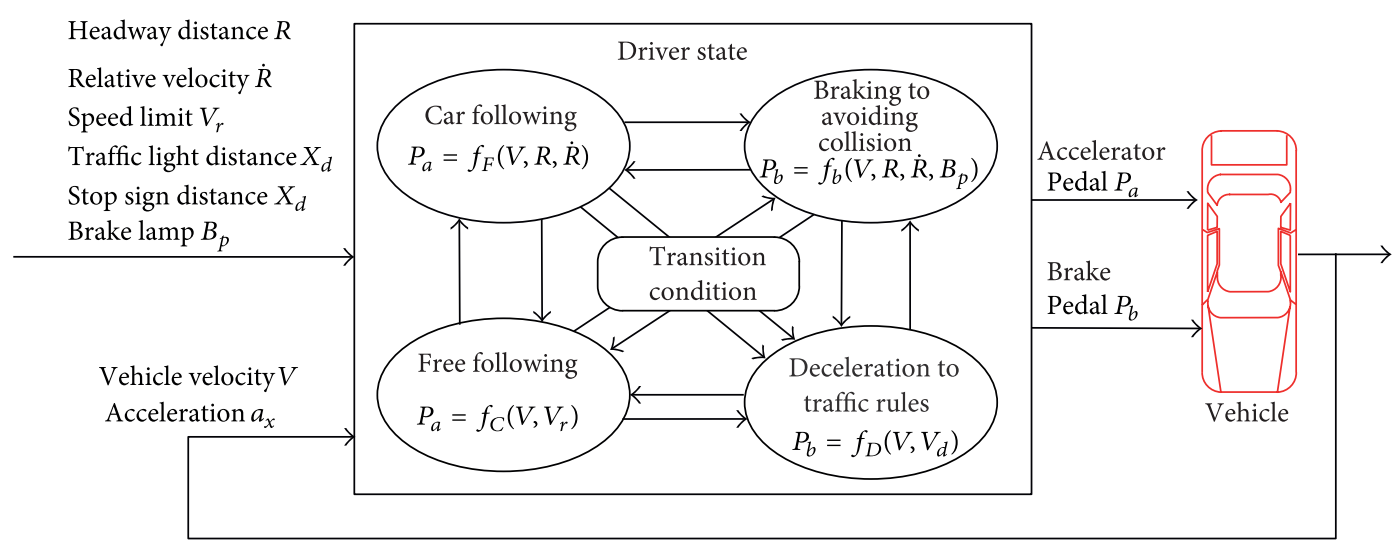

FIGURE 12: State flow diagram of the driver model with longitudinal driver-vehicle dynamics [79].

(e.g., the longitudinal velocity, acceleration, throttle/braking pedal pressure, and relative distance/velocity) collected under real traffic conditions. Time headway (THW) and time to collision (TTC) were then selected to analyze the longitudinal driving behavior. Subsequently, the parameters of the driver model were determined and identified in-time by using the recursive least-square (RLS) self-learning algorithm with a forgetting factor. Finally, an advanced automatic control systems (Figure 13) embedded with driver characteristics was designed and verified.

5.2. Lateral Control Based on Driver Characteristics. This subsection discusses lateral control systems based on driver characteristics. When the host vehicle is executing a following or tracking task on the straight way, the longitudinal control can be treated as the primary action to ensure that the distance between the host vehicle and the leading vehicle is safe enough. However, lateral control should be given great consideration in the following scenarios.

(1) The First Case Is Curve Driving. Before driving onto a curved road from a straight path or from one curved path to another, the driver usually decreases the vehicle's velocity before steering the wheel to track the curve road.

(2) The Second Is Lane Change. For instance, when a driver wants to overtake on the highway, lateral control is a vital for guaranteeing the host vehicle's safety in traffic flow.

(3) The Third Situation Is the Avoidance of Collisions. If the leading vehicle makes abrupt stop or if a person suddenly appears in front of a fast moving vehicle, the driver will most likely initiate some form of action to prevent a collision from happening, be it quickly steering the vehicle away or slamming on the brakes.

As seen from the abovementioned cases, steering wheel control is crucial for keeping the driver safe. In order to improve road tracking performance and relieve a driver's workload, the electric power steering (EPS) embedded with driver's characteristics has been designed. In $[84,85]$, the steering assistance systems (Figure 14) for driver characteristics are presented with two controllers (guidance and steering) designed based on the gain scheduled control theory by Fujiwara and Adachi.

In the steering assistance systems, $\Delta D$ depicts driver characteristics. The steering wheel angle $\theta_{S}$ and the vehicle's steering torque $T_{S}$ are considered as the characteristics of a driver's operation.

Driving an automobile can be considered as a closed-loop control task executed by the human driver [1]. By modeling and recognizing driver behavior based on driving data, the characteristics of human driving behavior/skill/state can be used for the advanced controller design of vehicle dynamics systems embedded with the driver's characteristics [79, 8385]. However, human driving behavior encompasses the characteristics of randomness and uncertainty; hence, traditional feedback control systems based on driver characteristics may not completely represent the driver-vehicle system. To accurately simulate the driver-vehicle systems as well as aid in the development of more advanced driver-vehicle control systems, other methods of advanced controller design might be adopted, such as the robust static output feedback control (SOF) $[86], H_{\infty}$ step tracking control [87], robust $H_{\infty}$ slidingmode control (SMC) [88], and the networked predictive control [89].

When driving from one curve onto another especially at high speeds, the vehicle might reach its handling limit and could lead to a rollover accident. With vehicle stability control as a key consideration, an intelligent personal minder (IPM) system (Figure 15) has been developed [90]. This vehicle control system contains an IPM system that is able to provide timely and clear advisory information to the driver. When certain parameter values of the vehicle are close to the vehicle's handling limit or the defining relative handling limit margin $h_{\text {env }}=\min \left\{h_{\mathrm{OS}}, h_{\mathrm{US}}, h_{\mathrm{TCS}}, h_{\mathrm{ABS}}, h_{\mathrm{SSRA}}\right\}$, the IPM will provide a timely warning to the driver, where $h_{\mathrm{OS}}$ and $h_{\mathrm{US}}$ are vehicle's yaw handling limit margin during oversteer and understeer situation respectively, $h_{\mathrm{TCS}}$ and $h_{\mathrm{ABS}}$ are the ABS and tracking handling limit margins, respectively, and $h_{\mathrm{SSRA}}$ is the vehicle's sideslip handling limit margin. According to the $h_{\text {env }}$, a driver's adaptive styles in handling the vehicle under 


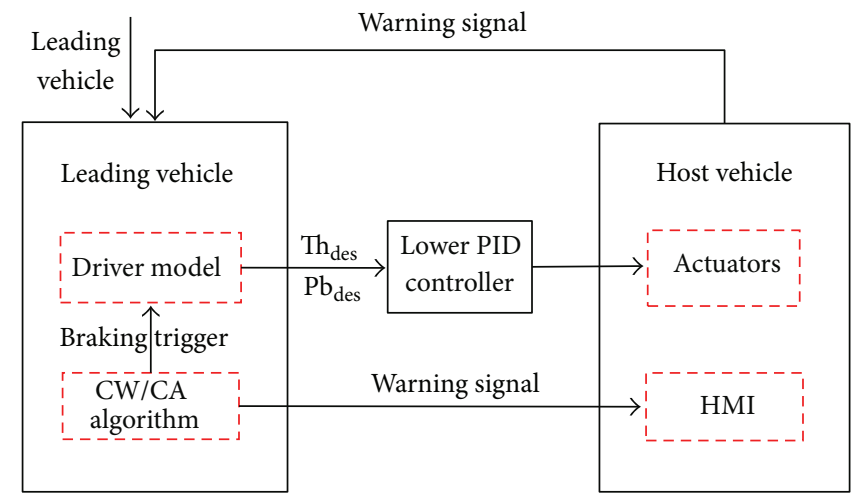

FIGURE 13: The control strategy of the driver assistance system [83].

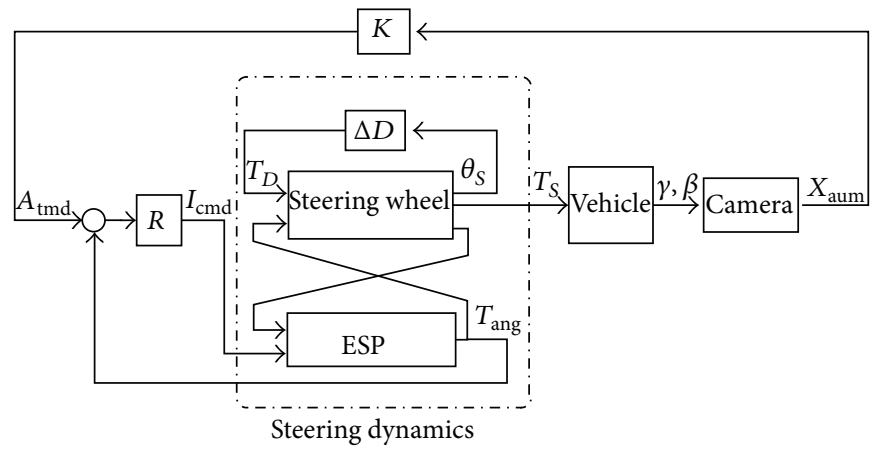

FIGURE 14: The system configuration of a driver-support-system embedded with driver characteristics [84, 85].

various driving conditions can be characterized in real-time with the control parameters adjusted correspondingly to the different driving styles; notwithstanding, this method should only be applied to long term rather than short term advisory. As driving skills can be used as a short term reference for adjusting the parameters of the DAS or ESC, it needs to be defined.

Similar to [91], for the purpose of improving the electronic system's intelligence and flexibility thus allowing it to better recognize the driver's expectations or intentions, three modes to characterize the driver's behavior or styles (aggressive and cautious behavior) were discussed based on the driver-in-the-loop system (Figure 16) established by Filev et al. [91].

(i) Characterizing Unstructured Driver Behavior. From the long-term perspective, longitudinal vehicle control might be primarily affected by the driver's behavior or driving styles, but not the vehicle dynamics response. It is usually conditionally applied to the unconstrained driver by detecting the brake pedal position or the rate change followed by making the corresponding adjustments to the variables of the vehicle; this is positive to the vehicle's fuel efficiency and acceleration performance. Statistical mathematics and probability theory are usually adopted in the process of characterizing unstructured driver behavior.

(ii) Characterizing Semistructured Driver Behavior. In this method, part signals of the electronic control systems can be used as the feedback information with the driving task being constrained, for example, car following, double lane change, and collision avoidance. The fuzzy control theory might be used in the course of recognizing driver behavior.

(iii) Characterizing Structured Driver Behavior. In order to fully utilize the vehicle's dynamic feedback information from the control theory point of view, the driving task should be described in more detail with the driver treated as a controller in the driver-in-the-loop system. To elaborate, the demanded safety distance between the host vehicle and the leading vehicle, the relative velocity of vehicles, and the TTC for car following might be used as the controller inputs. Since the driver models are structured under some fixed driving conditions, the classical control theory methods can be applied to the modeling or recognition driver behavior and skills.

Although numerous mathematical methods and concepts can be used to establish driver model to seamlessly coordinate driver and the electronic control system (ESC, DAS, ACC, IPM, etc.), further research needs to be made as there are still some issues left unaddressed.

(1) A System with Lower Order and Higher Accuracy. To design more intelligent and human-friendly vehicle's dynamic systems embedded with driver characteristics, driver model structures with greater accuracy but at the same time minimalistic and capable of both high-level cognitive processing and low-level operative control should be developed. 


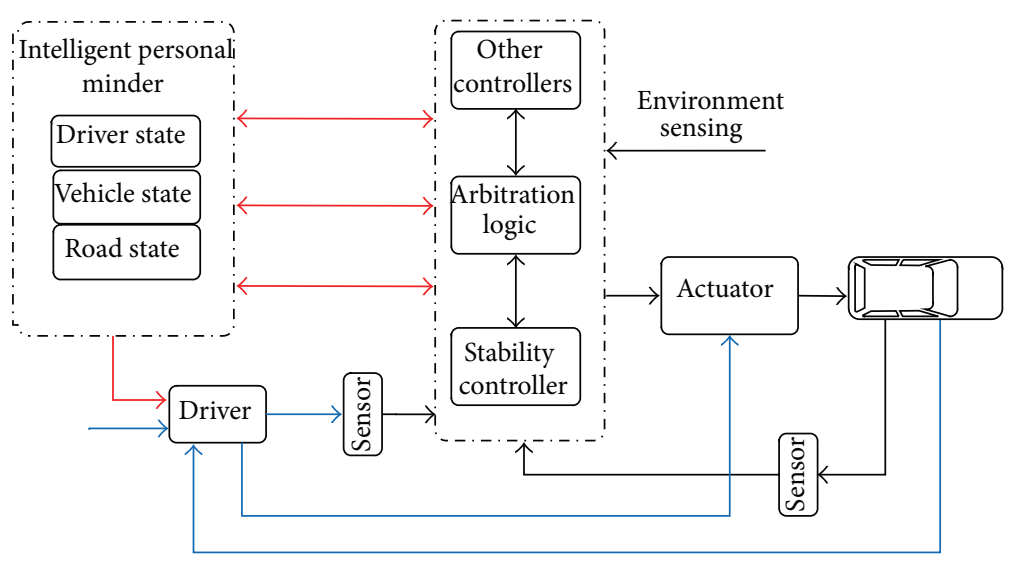

FIGURE 15: The block diagram of a vehicle control system augmented with an intelligent personal minder [90].

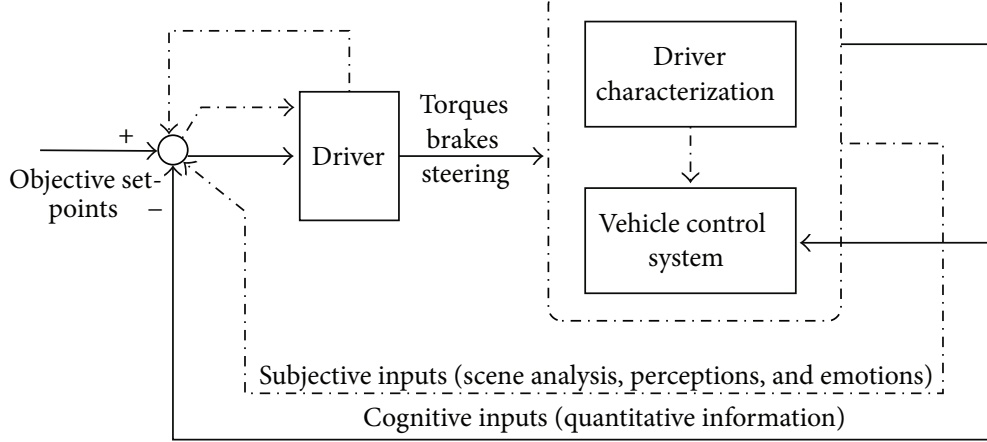

FIgURE 16: The driver-in-the-loop system configuration [91].

(2) Traffic Factors Should Be Considered. The various research studies we have discussed in this paper were only of humanvehicle interaction/cooperation and did not consider driving environment. When a driver is driving, the driver, the vehicle, and traffic factors can be treated as a closed-loop system, in which the traffic factors (e.g., weather condition, pedestrian behavior, the traffic light, and road conditions) have great influence on a driver's behavior. Therefore, we recommend that the relationships of vehicle to vehicle (V2V), vehicle to infrastructure (V2I), and vehicle to the external environment (V2E) to also be explored and studied further.

(3) Seamless Coordination between Vehicle and Driver Characteristics. The intelligence, reliability, and comfort provided by the seamless coordination between vehicle and driver characteristics can be improved on by introducing advanced mathematical methods, control theories, and system identification methods into the modeling and recognition of driver behavior and skills. Even though the concepts of "human-centered" and "driver-aware" vehicle systems have been proposed, the advanced ESC might impede on the driver's expectations and control due to inaccurate system identification as well as the influence of unstructured uncertainty models (e.g., additional uncertainty due to unmodeled dynamics).

\section{Conclusions}

The reviewed articles reveal a wide range of mathematical methods of modeling and recognition of driver characteristics which can be used to improve vehicle's dynamics performance, decrease driver's workload, and develop more intelligent driver assistance systems. Modeling and recognition of driver behavior/skill/state have great important significance in many fields, such as active safety systems, intelligence transport systems, and smart car systems. Most of the researches proposed in this paper have their focus on driver assistance systems and active safety systems, and their goals can be classified roughly as follows: (1) to put driver model in simulators with aim to evaluate and verify driver assistance systems; (2) to put driver model in simulators in order to recognize driver's driving behavior/skill/state in certain task (e.g., lane change, collision avoidance, and haste braking); (3) to be concern on about whether the human driver can fit to the vehicle systems or not by modeling and analyzing driver behavior; (4) to characterize driver's driving skill/state/styles with driver model.

This paper shows that numerous driver models have been developed from different perspectives by using various identification methods, and the characteristic parameters of driver's driving behavior/skill/state are not the same for 
different driving tasks and situations. Some methods may seem to be more efficient than others for certain driving tasks and situations described in this paper, but these methods might be not the best for other driving tasks. Therefore, some issues still exist, for example, which parameters and driving situations are more sensitive to driver's driving behavior/skill/state, that is, how to characterize the human driver more exactly, easily, and quickly? Hence, further research may be conducted as in the following aspects.

(1) The driver model which can accurately describe all the individual driver's behaviors for different driving tasks and situations should be developed.

(2) The advanced mathematical methods which can precisely and quickly characterize driver behavior and skill may be introduced and developed.

(3) The advanced control theory should be seamlessly coordinated with driver characteristics.

In addition, driver's psychological and physiological factors which are rarely discussed in this paper are crucial to driver models; hence, more and more researchers may show interests in modeling and recognition of driver behavior in the future.

\section{Conflict of Interests}

The authors declare that there is no conflict of interests regarding the publication of this paper.

\section{Acknowledgment}

This work was supported by the China Scholarship Council (CSC no. 2011603509).

\section{References}

[1] M. Plöchl and J. Edelmann, "Driver models in automobile dynamics application," Vehicle System Dynamics, vol. 45, no. 7-8, pp. 699-741, 2007.

[2] D. Oussama, P. Tamas, Z. Hossni, M. Benjamin, and B. Michel, "Modified intelligent driver model for driver safety and traffic stability improvement," in Proceedings of the 7th IFAC Symposium on Advances in Automotive Control (AAC '13), vol. 7, pp. 744-749, 2013.

[3] P. Carlo Cacciabue, Modelling Driver Behaviour in Automotive Environments, EC JRC-IPSC, 2007.

[4] Human Performance: Driver Behavior, Road Design and Intelligent Transportation Systems. Safety and Human Performance, TRB/TRR-1724, National Academy of Science Transportation Research Borad, Washington, DC, USA, 2000.

[5] J. Meech and J. Parreira, "An interactive simulation model of human drivers to study autonomous haulage trucks," in Proceedings of the Complex Adaptive Systems Conference, pp. 118-123, November 2011.

[6] H. A. Rakha, I. H. Zohdy, and R. K. Kamalanathsham, AgentBased Game Theory Modeling for Driverless Vehicles at Intersections, VT-2010-02, Department of Transportation, Research and Innovative Technology Administration (RITA), Washington, DC, USA, 2013.
[7] A. Liu and D. Salvucci, "Modeling and prediction of human driver behavior," in Proceedings of the 9th International Conference on Human-Computer Interaction, New Orleans, La, USA, 2001.

[8] Y. Zhang, W. C. Lin, and Y.-K. S. Chin, "A pattern-recognition approach for driving skill characterization," IEEE Transactions on Intelligent Transportation Systems, vol. 11, no. 4, pp. 905-916, 2010.

[9] A. Erséus, Driver-Vehicle Interaction, identification, characterization and modeling of path tracking skill [Ph.D. thesis], Royal Institute of Technology (KTH), Stockholm, Sweden, 2010.

[10] J. A. Groege, Understanding Driving: Applying Cognitive Psychology to a Complex Everyday Task, Psychology Press, 2000.

[11] H. Tokutake, Y. Sugimoto, and T. Shirakata, "Real-time identification method of driver model with steering manipulation," Vehicle System Dynamics, vol. 51, no. 1, pp. 109-121, 2013.

[12] P. Hermannstädter and B. Yang, "Identification and validation of lateral driver models on experimentally induced driving behavior," in Proceedings of the IEEE International Conference on Systems, Man, and Cybernetics, COEX, Seoul, Republic of Korea, October 2012.

[13] G. O. Burnham, J. Seo, and G. A. Bekey, "Identification of human driver models in car following," IEEE Transactions on Automatic Control, vol. AC-19, no. 6, pp. 911-915, 1974.

[14] M. Brackstone and M. McDonald, "Car-following: a historical review," Transportation Research F, vol. 2, no. 4, pp. 181-196, 1999.

[15] L.-K. Chen and A. G. Ulsoy, "Identification of a nonlinear driver model via NARMAX modeling," in Proceedings of the American Control Conference, pp. 2533-2537, Chicago, Ill, USA, June 2000.

[16] L.-K. Chen and A. G. Ulsoy, "Driver model uncertainty", in Proceedings of the American Control Conference (ACC '99), pp. 714-718, San Diego, Calif, USA, June 1999.

[17] L.-K. Chen and A. G. Ulsoy, "Identification of a driver steering model, and model uncertainty, from driving simulator data," Journal of Dynamic Systems, Measurement and Control, vol. 123, no. 4, pp. 623-629, 2001.

[18] C. C. Macadam, "Understanding and modeling the human driver," Vehicle System Dynamics, vol. 40, no. 1-3, pp. 101-134, 2003.

[19] H. Tsunai, K. Maeda, R. Hayashi, P. Raksineharoensak, and M. Nagai, "Development of steering behavior recognition method by using sensing data of drive recorder," in Proceedings of the International Conference on Control, Automation and Systems (ICCAS '08), pp. 301-306, October 2008.

[20] T. Pilutti and G. Ulsoy, "On-line identification of driver state for lane-keeping tasks," in Proceedings of the American Control Conference, pp. 678-681, June 1995.

[21] S. Sekizawa, S. Inagaki, T. Suzuki et al., "Modeling and recognition of driving behavior based on stochastic switched ARX model," IEEE Transactions on Intelligent Transportation Systems, vol. 8, no. 4, pp. 593-606, 2007.

[22] J. Bengtsson, R. Johansson, and A. Sjögren, "Modeling of drivers' longitudinal behavior," in Proceedings of the IEEUASME International Conference on Advanced Intelligent Mechatronics Proceedings, pp. 1076-1081, Como, Italy, July 2001.

[23] A. Reński, “Identification of driver model parameters," International Journal of Occupational Safety and Ergonomics, vol. 7, no. 1, pp. 79-90, 2001.

[24] L. Saleh, P. Chevrel, F. Mars, J.-F. Lafay, and F. Claveau, "Humanlike cybernetic driver model for lane keeping," in Proceedings of the 18th IFAC World Congress, pp. 4368-4373, Milano, Italy, 2011. 
[25] M. Mulder, M. Mulder, M. M. van Paassen, and D. A. Abbink, "Identification of driver car-following behaviour," in Proceedings of the International Conference on Systems, Man and Cybernetics, vol. 3, pp. 2905-2910, October 2005.

[26] J. Steen, H. J. Damveld, R. Happee, M. M. van Paassen, and M. Mulder, "A review of visual driver models for system identification purposes," in Proceedings of the IEEE International Conference on Systems, Man, and Cybernetics (SMC '11), pp. 2093-2100, October 2011.

[27] T. Wakita, K. Ozawa, C. Miyajima et al., "Driver identification using driving behavior signals," in Proceedings of the 8th International IEEE Conference on Intelligent Transportation Systems, pp. 907-912, Vienna, Austria, September 2005.

[28] T. Wakita, K. Ozawa, C. Miyajima, and K. Takeda, "Parametric versus non-parametric models of driving behavior signals for driver identification," in Proceedings of the 5th International Conference on Audio, and Video-Based Biometric Person Authentication (AVBPA '05), pp. 739-747, July 2005.

[29] C. Miyajima, Y. Nishiwaki, K. Ozawa, T. Wakita, K. Itou, and K. Takeda, "Cepstral analysis of driving behavioral signals for driver identification," in Proceedings of the IEEE International Conference on Acoustics, Speech and Signal Processing (ICASSP '06), pp. V921-V924, Toulouse, France, May 2006.

[30] D. Filev, J. Lu, F. Tseng, and K. Prakah-Asante, "Real-time driver characterization during car following using stochastic evolving models," in Proceedings of the IEEE International Conference on Systems, Man, and Cybernetics (SMC'11), pp. 1031-1036, October 2011.

[31] G. Nicola Bifulco, F. Galante, L. Pariota, and M. Russo-Spena, "Identification of driving behaviors with computer-aided tools," in Proceedings of the 6th European Modelling Symposium, pp. 331-336, 2012.

[32] W. Van Winsum and H. Godthelp, "Speed choice and steering behavior in curve driving," Human Factors, vol. 38, no. 3, pp. 434-441, 1996.

[33] A. M. C. Odhams and D. J. Cole, Models of Driver Speed Choice in Curves, 2004.

[34] A. B. Ellison and S. Greaves, "Driver characteristics and speeding behaviour," in Proceedings of the 33rd Australasian Transport Research Forum (ATRF '10), 2010.

[35] P. Angkititrakul, C. Miyajima, and K. Takeda, "Modeling and adaptation of stochastic driver-behavior model with application to car following," in Proceedings of the IEEE Intelligent Vehicles Symposium (IV '11), pp. 814-819, Baden-Baden, Germany, June 2011.

[36] P. Angkititrakul, C. Miyajima, and K. Takeda, "An improved driver-behavior model with combined individual and general driving characteristics," in Proceedings of the IEEE Intelligent Vehicles Symposium (IV '12), pp. 426-431, Alcalá de Henares, Spain, 2012.

[37] W. C. Lin, Y.-K. Chin, B. S. Repa, M. Lu, R. L. Nisonger, and C.G. Liang, "Characterisation of driving skill level using driving simulator tests," International Journal of Vehicle Autonomous Systems, vol. 5, no. 3-4, pp. 219-229, 2007.

[38] T. Pilutti and A. Galip Ulsoy, "Identification of driver state for lane-keeping tasks," IEEE Transactions on Systems, Man, and Cybernetics A, vol. 29, no. 5, pp. 486-502, 1999.

[39] T. Pilutti and A. G. Ulsoy, "Identification of driver state for lane-keeping tasks: experimental results," in Proceedings of the American Control Conference, pp. 3370-3374, June 1997.

[40] K. Abe, H. Miyatake, and K. Oguri, "A study on switching ARHMM driving behavior model depending on driver's states," in
Proceedings of the 10th International IEEE Conference on Intelligent Transportation Systems (ITSC '07), pp. 806-811, October 2007.

[41] P. Boyraz, M. Acar, and D. Kerr, "Signal modelling and Hidden Markov models for driving manoeuvre recognition and driver fault diagnosis in an urban road scenario," in Proceedings of the IEEE Intelligent Vehicles Symposium (IV '07), pp. 987-992, June 2007.

[42] A. Sathyanarayana, P. Boyraz, and J. H. L. Hansen, "Driver behavior analysis and route recognition by hidden Markov models," in Proceedings of the IEEE International Conference on Vehicular Electronics and Safety (ICVES '08), pp. 276-281, September 2008.

[43] A. Pentland and L. Andrew, "Modeling and prediction of human behavior," Neural Computation, vol. 11, no. 1, pp. 229242, 1999.

[44] M. C. Nechyba and Y. Xu, "On the fidelity of human skill models," in Proceedings of the 13th IEEE International Conference on Robotics and Automation, pp. 2688-2693, April 1996.

[45] N. Kuge, T. Yamamura, O. Shimoyama, and A. Liu, "A driver behavior recognition method based on a driver model framework," SAE Technical Paper Series 2000-01-0349, 2000.

[46] J.-H. Kim, S. Okuma, Y.-W. Kim, D.-H. Hwang, M.-H. Kim, and D.-H. Kim, "Modeling of human driving behavior based on piecewise linear model," in Proceedings of the IEEE International Symposium on Industrial Electronics (ISIE '05), pp. 25-30, Dubrovnik, Croatia, June 2005.

[47] J.-H. Kim, S. Hayakawa, T. Suzuki et al., "Modeling of driver's collision avoidance maneuver based on controller switching model," IEEE Transactions on Systems, Man, and Cybernetics B, vol. 35, no. 6, pp. 1131-1143, 2005.

[48] J.-H. Kim, S. Hayakawa, T. Suzuki et al., "Modeling of driver's collision avoidance behavior based on piecewise linear model," in Proceedings of the 43rd IEEE Conference on Decision and Control (CDC '04), pp. 2310-2315, December 2004.

[49] J. A. Michon, "A critical view of driver behavior models: what do we know, what should we do?" in Behavior and Traffic Safety, pp. 485-520, 1985.

[50] U. Kiencke, R. Majjad, and H. KoKrner, "Design of a hybrid driver model," SAE Paper 980017, International Congress and Exposition, Detroit, Mich, USA, 1998.

[51] T. Akita, T. Suzuki, S. Hayakawa, and S. Inagaki, "Analysis and synthesis of driving behavior based on mode segmentation," in Proceedings of the International Conference on Control, Automation and Systems (ICCAS '08), pp. 2884-2889, Seoul, Republic of Korea, October 2008.

[52] M. Okamoto, S. Otani, Y. Kaitani, and K. Uchida, "Identification of driver operations with extraction of driving primitives," in Proceedings of the 20th IEEE International Conference on Control Applications (CCA '11), pp. 338-344, September 2011.

[53] Y. Fujiwara, T. Fujihira, O. S. Ishiwa, and S. Adachi, "Control design of driver support system using multiple driver models," in Proceedings of the SICE Annual Conference, pp. 2773-2778, Hokkaido Institute of Tecnology, Sapporo, Japan, August 2004.

[54] U. Kiencke, R. Majjad, and S. Kramer, "Modeling and performance analysis of a hybrid driver model," Control Engineering Practice, vol. 7, no. 8, pp. 985-991, 1999.

[55] B. Song, D. Delorme, and J. VanderWerf, "Cognitive and hybrid model of human driver," in Proceedings of the IEEE Intelligent Vehicles Symposium, pp. 1-6, Dearborn, Mich, USA, October 2000. 
[56] T. Suzuki, S. Yamada, S. Hayakawa, N. Tsuchida, T. Tsuda, and H. Fujinami, "Modeling of drivers collision avoidance behavior based on hybrid system model: an approach with data clustering," in Proceedings of the International Conference on Systems, Man and Cybernetics, pp. 3817-3822, October 2005.

[57] T. Akita, S. Inagaki, T. Suzuki, S. Hayakawa, and N. Tsuchida, "Analysis of vehicle following behavior of human driver based on hybrid dynamical system model," in Proceedings of the 16th IEEE International Conference on Control Applications, pp. 1233-1238, Singapore, October 2007.

[58] T. Akita, S. Inagaki, T. Suzuki, S. Hayakawa, and N. Tsuchida, "Hybrid system modeling of human driver in the vehicle following task," in Proceedings of the SICE Annual Conference (SICE '07), pp. 1122-1127, Kagawa University, September 2007.

[59] A. Kurt, J. L. Yester, Y. Mochizuki, and Ü. Özgüner, "Hybridstate driver/vehicle modelling, estimation and prediction," in Proceedings of the 13th International IEEE Conference on Intelligent Transportation Systems (ITSC '10), pp. 806-811, Madeira Island, Portugal, September 2010.

[60] M. Mukai and T. Kawabe, "Model predictive control for lane change decision assist system using hybrid system representation," in Proceedings of the SICE-ICASE International Joint Conference, pp. 5120-5125, Bexco, Busan, Republic of Korea, October 2006.

[61] H. Okuda, N. Ikami, T. Suzuki, Y. Tazaki, and K. Takeda, "Modeling and analysis of driving behavior based on a probabilityweighted ARX model," IEEE Transactions on Intelligent Transportation Systems, vol. 14, no. 1, pp. 98-112, 2013.

[62] M. Shangchang, T. Zanma, and M. Ishida, "Identification of human skill and its application to an automatic driving system: an approach from hybrid dynamical system," in Proceedings of the 32nd Annual Conference on IEEE Industrial Electronics, pp. 400-405, November 2006.

[63] S. Ma, B. Yang, Y. Zhan, S. Zhang, and C. Wang, "Automatic driving system based on embedded microcontroller using human skill models," Journal of Computers, vol. 6, no. 2, pp. 351358, 2011.

[64] T. Hsiao, "Time-varying system identification via maximum a posteriori estimation and its application to driver steering models," in Proceedings of the American Control Conference (ACC '08), pp. 684-689, Washington, DC, USA, June 2008.

[65] R. P. Harper, Pilot Evaluation of Handling Qualities, Cornell Aeronautical Lab Inc, Buffalo, NY, USA, 1965.

[66] A. Modjtahedzadeh and R. A. Hess, "Model of driver steering dynamics for use in assessing vehicle handling qualities," in Proceedings of the Winter Annual Meeting of the American Society of Mechanical Engineers, vol. 40, pp. 41-56, December 1991.

[67] D. S. Carlo, P. Leonardo, and C. Santi, "Handling objective evaluation using a parametric driver model for ISO lane change simulation," in Proceedings of the SAE Automotive Dynamics and Stability Conference and Exhibition, May 2002.

[68] S. Horiuchi and N. Yuhara, "An analytical approach to the prediction of handling qualities of vehicles with advanced steering control system using multi-input driver model," Journal of Dynamic Systems, Measurement and Control, vol. 122, no. 3, pp. 490-497, 2000.

[69] Y. Miura, H. Tokutake, and K. Fukui, "Handling qualities evaluation method based on actual driver characteristics," Vehicle System Dynamics, vol. 45, no. 9, pp. 807-817, 2007.
[70] J. Ishio, H. Ichikawa, Y. Kano, and M. Abe, "Vehiclehandling quality evaluation through model-based driver steering behaviour," Vehicle System Dynamics, vol. 46, no. 1, pp. 549560, 2008.

[71] H. Peng, "Evaluation of driver assistance systems: a human 28 centered approach," in Proceedings of the 6th International Symposium on Advanced Vehicle Control (AVEC '02), Hiroshima, Japan, September 2002.

[72] K. Lee and H. Peng, "Identification of a longitudinal human driving model for adaptive cruise control performance assessment," in Proceedings of the International Mechanical Engineering Congress and Exposition (IMECE '02), pp. 675-682, November 2002.

[73] H.-H. Yang and H. Peng, "Development of an errorable carfollowing driver model," Vehicle System Dynamics, vol. 48, no. 6, pp. 751-773, 2010.

[74] H.-H. Yang and H. Peng, "Development and evaluation of collision warning/collision avoidance algorithms using an errable driver model," Vehicle System Dynamics, vol. 48, no. 1, pp. 525535, 2010.

[75] P. Fancher, Z. Bareket, and R. Ervin, "Human-centered design of an Acc-with-braking and forward-crash-warning system," Vehicle System Dynamics, vol. 36, no. 2-3, pp. 203-224, 2001.

[76] A. M. Vadeby, "Modeling of relative collision safety including driver characteristics," Accident Analysis and Prevention, vol. 36, no. 5, pp. 909-917, 2004.

[77] M. A. Goodrich and E. R. Boer, "Designing human-centered automation: tradeoffs in collision avoidance system design," IEEE Transactions on Intelligent Transportation Systems, vol. 1, no. 1, pp. 40-54, 2000.

[78] J. C. McCall, O. Achler, and M. M. Trivedi, "Design of an instrumented vehicle test bed for developing a human centered driver support system," in Proceedings of the IEEE Intelligent Vehicles Symposium, pp. 483-488, University of Parma, Parma, Italy, June 2004.

[79] P. Raksincharoensak, W. Khaisongkram, M. Nagai, M. Shimosaka, T. Mori, and T. Sato, "Integrated driver modelling considering state transition feature for individual adaptation of driver assistance systems," Vehicle System Dynamics, vol. 48, no. 1, pp. 55-71, 2010.

[80] J. C. McCall, O. Achler, M. M. Trivedi et al., "A collaborative approach for human-centered driver assistance systems," in Proceedings of the 7th International IEEE Conference on Intelligent Transportation Systems (ITSC '04), pp. 663-667, Washington, DC, USA, October 2004.

[81] P. Zheng and M. McDonald, "Manual versus adaptive cruise control: can driver's expectation be matched?" Transportation Research C, vol. 13, no. 5-6, pp. 421-431, 2005.

[82] M. Nakaoka, P. Raksincharoensal, and M. Nagai, "Study on forward collision warning system adapted to driver characteristics and road environment," in Proceedings of the International Conference on Control, Automation and Systems (ICCAS '08), pp. 2890-2895, COEX, Seoul, Korea, October 2008.

[83] J. Wang, L. Zhang, D. Zhang, and K. Li, "An adaptive longitudinal driving assistance system based on driver characteristics," Transactions on Intelligent Transportation Systems, vol. 14, no. 1, pp. 1-112, 2013.

[84] Y. Fujiwara and S. Adachi, "Control design of steering assistance system for driver characteristics," in Proceedings of the 41st SICE Annual Conference (SICE '02), Osaka, Japan, August 2002.

[85] Y. Fujiwara and S. Adachi, "Steering assistance system for driver characteristics using gain scheduling control," in Proceedings 
of the European Control Conference (ECC '03), pp. 3622-3627, Cambridge, UK, September 2003.

[86] H. Zhang, Y. Shi, and A. Saadat Mehr, "Robust static output feedback control and remote PID design for networked motor systems," IEEE Transactions on Industrial Electronics, vol. 58, no. 12, pp. 5396-5405, 2011.

[87] H. Zhang, Y. Shi, and M. Liu, " $H_{\infty}$ step tracking control for networked discrete-time nonlinear systems with integral and predictive actions," IEEE Transactions on Industrial Informatics, vol. 9, no. 1, pp. 337-345, 2013.

[88] H. Zhang, J. Wang, and Y. Shi, "Robust $H_{\infty}$ sliding-mode control for Markovian jump systems subject to intermittent observations and partially known transition probabilities," Systems \& Control Letters, vol. 62, no. 12, pp. 1114-1124, 2013.

[89] H. Zhang, Y. Shi, and J. Wang, "Observer-based tracking controller design for networked predictive control systems with uncertain Markov delays," International Journal of Control, vol. 86, no. 10, pp. 1824-1836, 2013.

[90] J. Lu, D. Filev, K. P. Asante, F. Tseng, and I. V. Kolmanovsky, "From vehicle stability control to intelligent personal minder: real-time vehicle handling limit warning and driver style characterization," in Proceedings of the IEEE Workshop on Computational Intelligence in Vehicles and Vehicular Systems (CIVVS '09), pp. 43-50, April 2009.

[91] D. Filev, J. Lu, K. Prakah-Asante, and F. Tseng, "Real-time driving behavior identification based on driver-in-the-loop vehicle dynamics and control," in Proceedings of the IEEE International Conference on Systems, Man and Cybernetics (SMC '09), pp. 2020-2025, October 2009. 


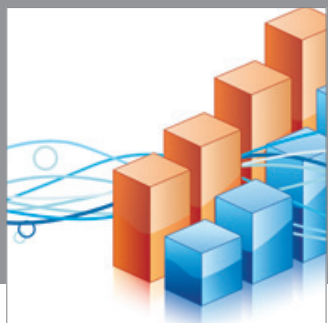

Advances in

Operations Research

mansans

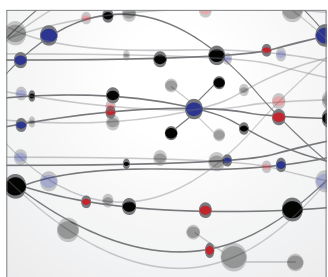

The Scientific World Journal
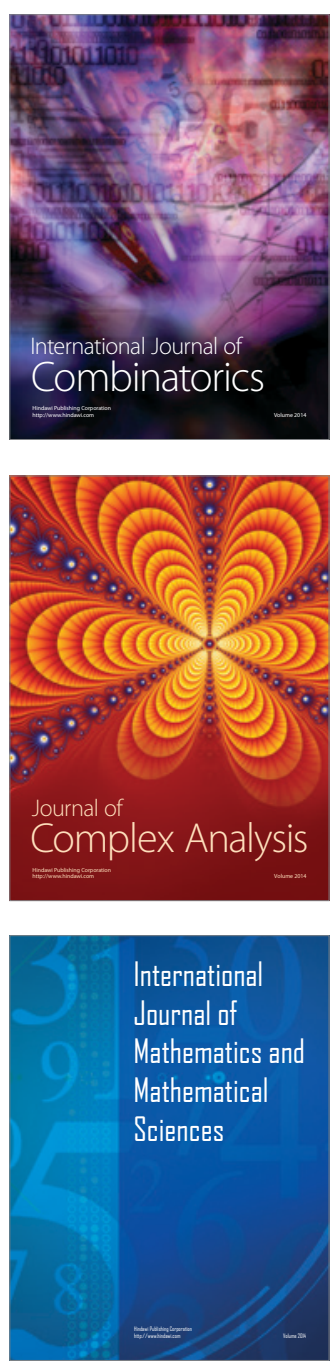
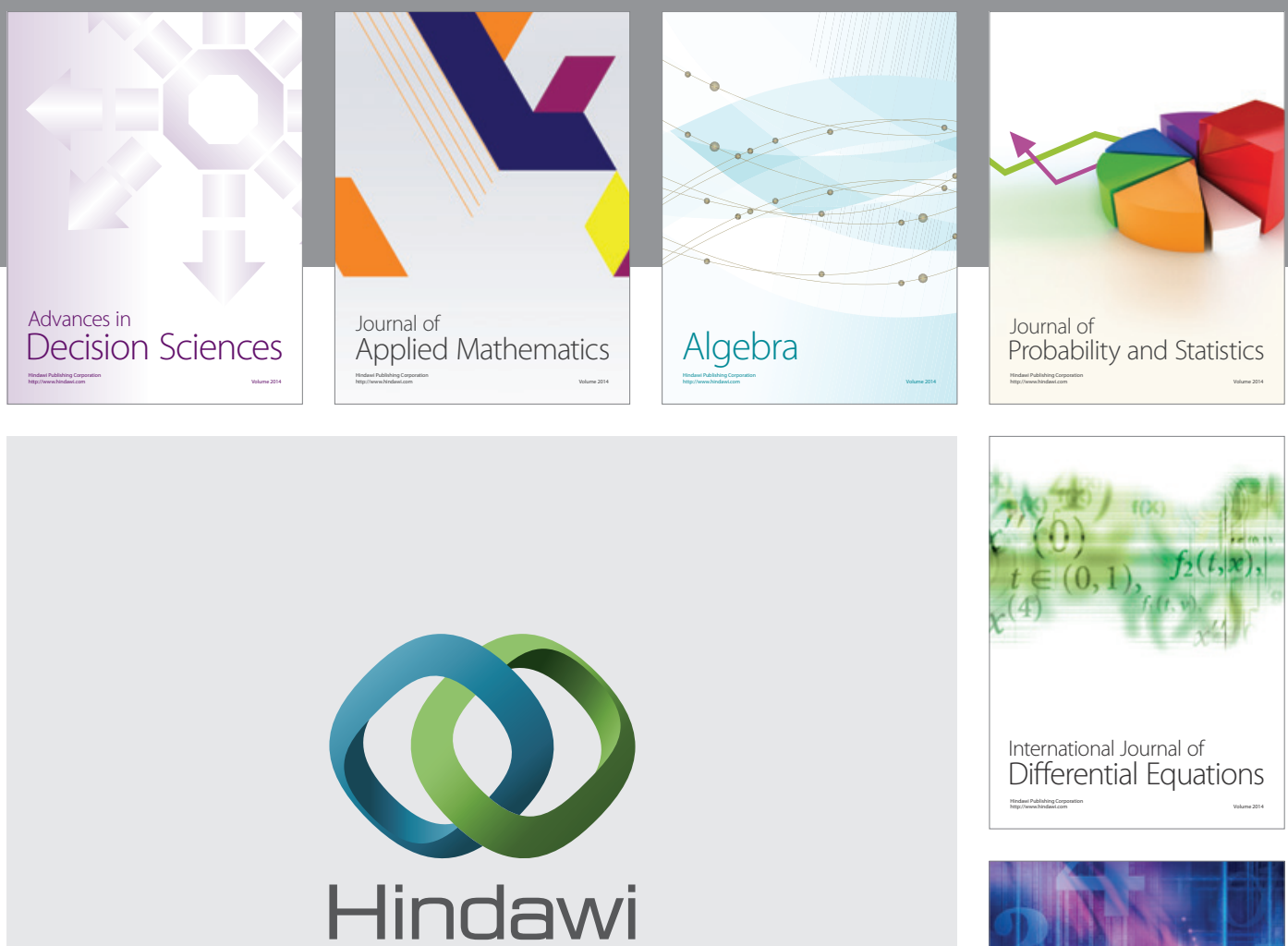

Submit your manuscripts at http://www.hindawi.com
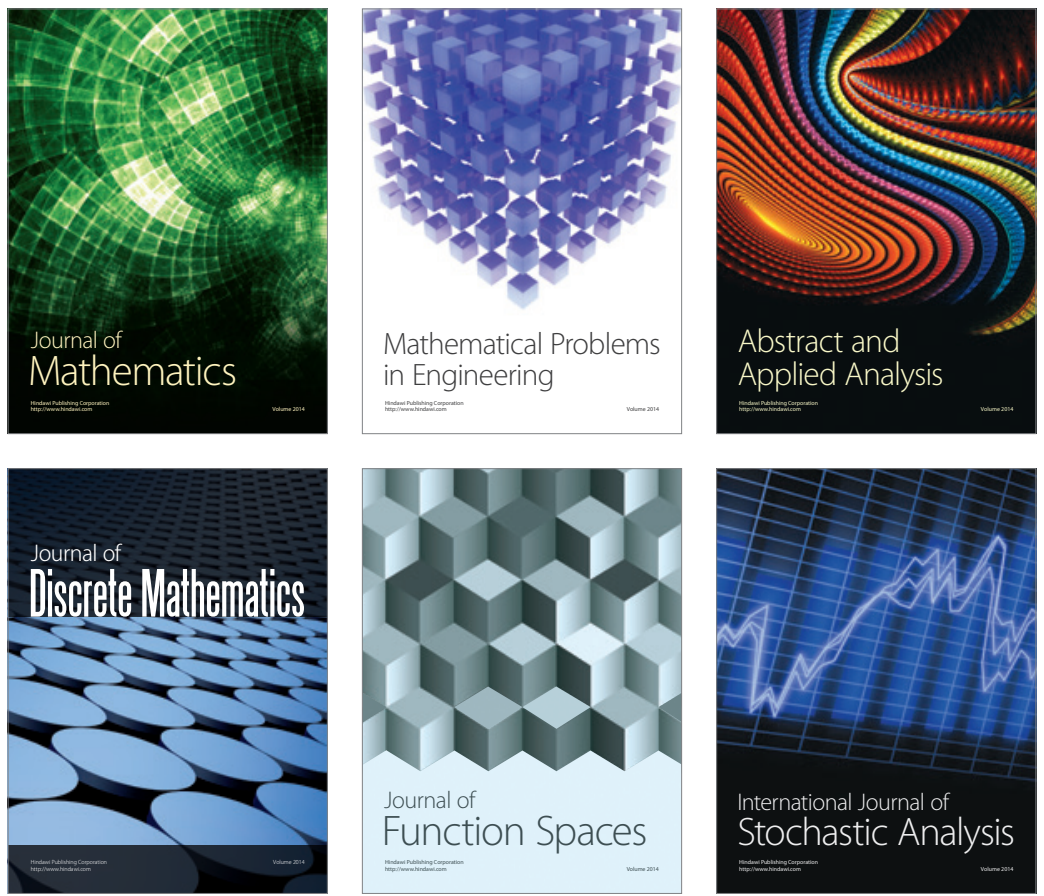

Journal of

Function Spaces

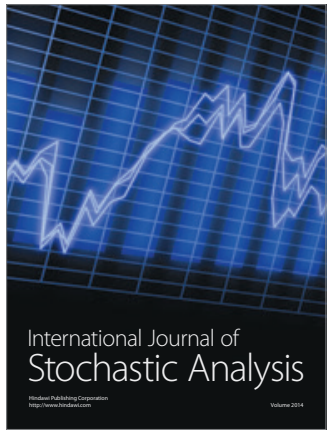

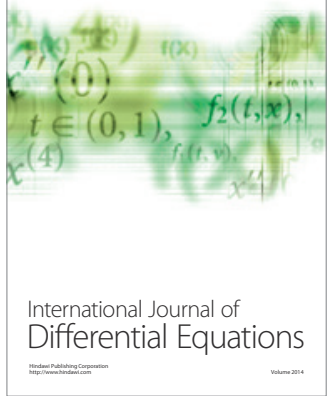
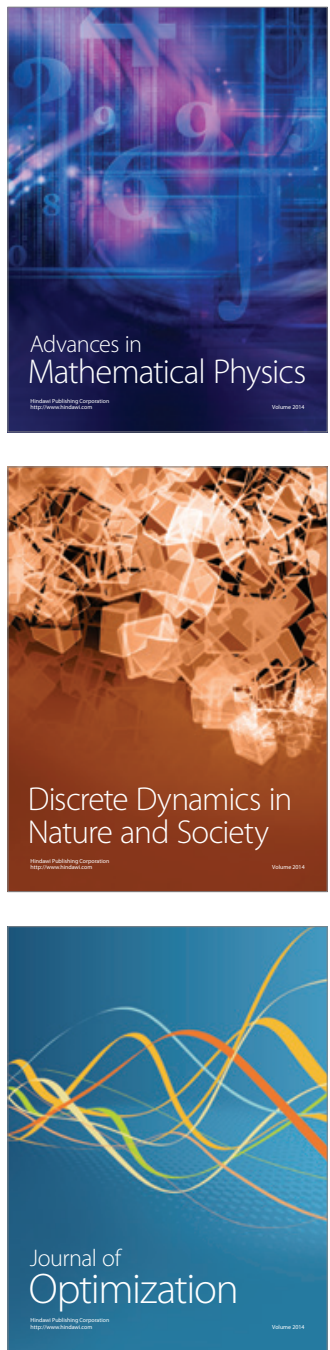